\title{
Cone Signals in Monostratified and Bistratified Amacrine Cells of Adult Zebrafish Retina
}

\author{
M.M. Torvund ${ }^{1,5}$, T.S. Ma ${ }^{1,6}$, V.P. Connaughton ${ }^{2}$, F. Ono ${ }^{3,4}$, and R.F. Nelson ${ }^{1,{ }^{*}}$ \\ ${ }^{1}$ National Institute of Neurological Disorders and Stroke, National Institutes of Health, Rockville \\ Maryland 20892 \\ ²Biology Department, American University, Washington, DC 20016 \\ ${ }^{3}$ National Institute of Alcoholism and Alcohol Abuse, National Institutes of Health, Rockville \\ Maryland 20892 \\ ${ }^{4}$ Department of Physiology, Osaka Medical College, Takatsuki, Japan 569-8686 \\ ${ }^{5}$ Graduate Program in Neuroscience, University of Arizona, Tucson, Arizona 85421 \\ ${ }^{6}$ University of Pennsylvania, Department of Neurosurgery, Philadelphia, PA 19104
}

\begin{abstract}
Strata within the inner plexiform layer (IPL) of vertebrate retinas are suspected to be distinct signaling regions. Functions performed within adult zebrafish IPL strata were examined through microelectrode recording and staining of stratified amacrine types. The stimulus protocol and analysis discriminated the pattern of input from red, green, blue, and UV cones as well as the light-response waveforms in this tetrachromatic species. A total of 36 cells were analyzed. Transient depolarizing waveforms at $\mathrm{ON}$ and OFF originated with bistratified amacrine types, whose dendritic planes branched either in IPL sublaminas $a \& b$, or only within sublamina $a$. Monophasic-sustained depolarizing waveforms originated with types monostratified in IPL s4 (sublamina $b$ ). OFF responses hyperpolarized at onset, depolarized at offset, and in some cases depolarized during mid-stimulus. These signals originated with types monostratified in s1 or s2 (sublamina a). Bistratified amacrines received depolarizing signals only from red cones, at both $\mathrm{ON}$ and OFF, while s4 stratified ON cells combined red and green cone signals. The s1/s2 stratified OFF cells utilized hyperpolarizing signals from red, red and green, or red and blue cones at $\mathrm{ON}$, but only depolarizing red cone signals at OFF. ON and OFF depolarizing transients from red cones appear widely distributed within IPL strata. "C-type" physiologies, depolarized by some wavelengths, hyperpolarized by others, in biphasic or triphasic spectral patterns, originated with
\end{abstract}

"CORRESPONDENCE TO: Ralph F. Nelson, National Institutes of Health, 5625 Fisher's Lane, Room 4S26, Rockville MD 20892. nelsonr@ninds.nih.gov.

CONFLICT OF INTEREST STATEMENT

None of the authors has any conflict of interest.

ROLE OF AUTHORS

MMT and RFN had full access to all the data in the study and take responsibility for the integrity of the data and the accuracy of the data analysis. Study concept and design: MMT, FO, RN. Acquisition of data: MMT, TSM, VPC, RN. Enhancer Trap: FO. Transgenic screening of retinas: VPC, FO, RFN; Analysis and interpretation of data: MMT, RN. Drafting of the article, statistical analysis, administration, supervision, and funding: RN. Critical revisions: all authors. 
amacrine cells monostratified in s5. Collectively, cells in this stratum processed signals from all cone types.

\section{INDEXING TERMS}

wavelength; 3D-reconstruction; spectral model; inner plexiform layer; GE4a transgenic; enhancer trap; ON-OFF response; ON response; OFF response; RRID: SCR_003070; RRID: SCR_011323; RRID: SCR_001775; RRID: SCR_002368; RRID: SCR_008593; RRID: SCR_008597; RRID: SCR_008406; RRID: SCR_013672; RRID: SCR_008960; RRID: SCR_013318; RRID: SCR_002798

\section{Cyprinids: an historical contribution to color vision}

In the 1960s, neuroscientists turned to cyprinid fish to search for the neural substrate of color vision. Laboratory mammals such as cat, rat, mouse, and rabbit, while providing outstanding models of visual pathways, seemed to lack a color sense. Alone in the mammalian kingdom, old world primates, including humans, saw trichromatic color. However, in goldfish (Wagner et al., 1960), as in primates (Gouras, 1968), retinal ganglion cells were wavelength selective, excited by some wavelengths and inhibited by others, correlating with behavioral evidence of color perception in both species. The existence of color-opponent ganglion cells lent support to an "opponent" theory of color vision, in which signals from cones with differently peaking opsin absorbances subtract to enhance wavelength acuity.

\section{Zebrafish: a color vision model with transgenes}

Zebrafish is a tetrachromatic cyprinid with color discrimination (Krauss and Neumeyer, 2003; Colwill et al., 2005; Orger and Baier, 2005; Avdesh et al., 2012). Zebrafish opsin genes and opsin spectral properties are well characterized (Hughes et al., 1998; Chinen et al., 2003; Endeman et al., 2013). There are four cone types: the principal and accessory members of double cones, long singles, and short singles (Engstrom, 1960). Principal cones are red cones, expressing long-wavelength-sensitive opsins (LWS1, LWS2); accessory cones are green cones expressing middle-wavelength-sensitive opsins (RH2-1, RH2-2, RH2-3, RH2-4); long singles are blue cones expressing short-wavelength-sensitive opsin (SWS2); and short singles are UV cones expressing UV-sensitive opsin (SWS1) (Raymond et al., 1995; Chinen et al., 2003). In the retinal outer plexiform layer of zebrafish, horizontal cells selectively contact cone types ( $\mathrm{Li}$ et al., 2009) and show evidence of opponent color signals, including biphasic, triphasic, and tetraphasic spectral patterns (Connaughton and Nelson, 2010). Potentially then, zebrafish is a model for vertebrate color processing mechanisms.

Bipolar cells relay cone signals from the outer plexiform layer to the inner plexiform layer (IPL). In zebrafish, some of the selective cone connectivity of bipolar cells in the outer retina, as well as the axonal layering of bipolar cell axons in the inner retina, has been studied (Li et al., 2012). Physiological evidence for color processing in the inner retina is seen in the zebrafish proximal negative response. This extracellular field potential contains narrow-field signals for red-green color contrast (Burkhardt, 2012). 


\section{Functional roles of amacrine cells}

Early reports of amacrine physiology found transient excitation at light onset and offset, the ON-OFF response (Dowling and Werblin, 1969; Kaneko, 1973). This led to the theory that the outer retina processes space and color, and the inner retina processes change and motion. Mammalian cholinergic amacrine cells (Tauchi and Masland, 1984), with intimate involvement in detection of directional movement, fit into the "motion processing" pattern (Barlow and Levick, 1965; Euler et al., 2002; Briggman et al., 2011).

Amacrine cells are anatomically quite diverse (Kolb et al., 1981; Wagner and Wagner, 1988; MacNeil et al., 1999; Jusuf and Harris, 2009). A major goal has been to find the physiological correlates of this diversity. Some amacrine morphologies target particular photoreceptor signals. For example, in mammals, the circuitry of two very significant amacrine cell populations, the AII and the A17, receive ribbon contacts mainly from rod bipolar cells and, physiologically, process mainly rod signals (Kolb and Famigilietti, 1974; Kolb, 1979; Nelson, 1982; Nelson and Kolb, 1985). These rod circuitry cells, like many amacrine cells, react to change in stimulation; but they specialize in doing this in the dark (Kolb and Famigilietti, 1974). A blue-cone-selective amacrine type (SCA) has recently been discovered in ground squirrel retina (Chen and $\mathrm{Li}, 2012)$. This cell only contacts bipolar cells that themselves are selective for blue or short-wavelength-sensitive (SWS) cones. Amacrines AII and SCA are both broadly bistratified, with bipolar-cell synaptic input in the proximal IPL sublamina $b$, and with either implied or demonstrated glycinergic inhibitory synaptic output onto ganglion-cell center mechanisms (Kolb, 1979; Müller et al., 1988; Sher and DeVries, 2012). A similar inhibitory blue-cone-selective amacrine cell has been proposed in rabbit retina to drive the "inverted S-OFF" color-opponent ganglion cell (Mills et al., 2014). While the AII cell, the A17 cell, and the SCA cell process particular opsin signals selectively, neither is color opponent, and no mammalian amacrine cells are yet known to be color opponent.

Some cyprinid amacrine cells are color opponent. One sustained type, described by Kaneko (1973), was hyperpolarized by red stimuli but depolarized by green. Further studies reveal not only red-green but yellow-blue opponency in carp (Mitarai et al., 1978). Coloropponent amacrine cells are a component of turtle retina neural circuits. Mainly these are red OFF, blue ON types (Ammermüller et al., 1995). In nonmammals, amacrine cells actively recombine signals arising from cones with different opsin types.

\section{Stratification patterns of amacrine cells}

Amacrine cells may be monostratified, bistratified, or diffuse. Amacrine dendritic branching patterns restrict the availability of synaptic partners with which to perform temporal and spectral processing. The IPL of mammals is divided into five strata; the IPL of zebrafish is divided into six (Connaughton et al., 2004). Strata are thought to perform selective functions. As examples, stratum s5 in mammals is the plane in which rodbipolar-cell or blue-cone-bipolar-cell axon terminals synapse on $A I I$ or SCA amacrine cells; stratum s1 in mammals is the plane of termination for $A I I$ synapses on OFF-type ganglion cells (Kolb, 1979). Dendritic field size is another restriction on synaptic connectivity. Finally, of course, 
the molecules of synaptic adhesion determine which of locally possible synapses actually form (Lewis et al., 2011). The physiological properties of amacrine light responses are determined by synaptic partners, and it is a common finding that dendritic form and function are closely linked in amacrine cells. Therefore it is reasonable that stratification pattern and dendritic extent are the basic indices for amacrine cell classification (Kolb et al., 1981; Wagner and Wagner, 1988). In both adult (Connaughton et al., 2004) and larval (Jusuf and Harris, 2009) zebrafish, amacrine types have been named according to these metrics.

\section{Statement of research goals}

The overall goal is to determine the functional structure of zebrafish inner retina, and to place this structure within the realm of vertebrate models. Here we penetrate zebrafish amacrine cells with stain-filled microelectrodes to correlate light responses with dendritic branching patterns. The light responses are categorized according to response waveform dynamics and also according to the patterns of input from the red, green, blue, or UV cones. The latter are inferred from a spectral model that represents the response dataset in terms of cone signal input. Both cone selective and cone opponent signals are detected within the datasets. Using a forward-transgenic line where IPL boundaries are marked by select populations of green fluorescent protein (GFP)-expressing amacrine and ganglion cells, the dendritic stratification patterns of microelectrode-injected amacrine cells are reconstructed in Neurolucida for position within the IPL. Cells are grouped according to physiological properties, and the correlation of amacrine cell spectral and temporal waveform properties with dendritic stratification is examined. In this way some of the physiological functions performed within zebrafish IPL strata can be inferred.

\section{MATERIALS AND METHODS}

\section{Maintenance of zebrafish lines for microelectrode studies}

Zebrafish were maintained in a stand-alone, recirculating, Aquatic Habitats benchtop system (http://pentairaes.com/aquatic-habitats, RRID:SCR_008597), following a holding and breeding protocol approved by the National Institute of Neurological Disorders and Stroke/ National Institute on Deafness and Other Communication Disorders IACUC (ASP 1307, ASP 1227). Wild-type (TL) and transgenic (GE4a) adult zebrafish were imported from the Laboratory of Molecular Physiology, National Institute on Alcohol Abuse and Alcoholism and the Unit on Behavioral Neurogenetics, National Institute of Child Health and Human Development. Transgenic fish were spawned, phenotyped by fluorescent protein expression at 3 days post fertilization (dpf), and reared to adulthood. Adult fish (male or female, 12-20 months old) were used in microelectrode studies.

\section{Generation of the GE4a transgenic zebrafish}

Zebrafish were maintained as above, but following the guidelines of either the University of Florida (ASP D464) or the National Institute on Alcohol Abuse and Alcoholism (ASP LMPFO-11). Using an enhancer trap method (Kawakami et al., 2004), transposase and a DNA construct containing the Hsp70 promoter (Halloran et al., 2000), as well as the enhanced (e)GFP gene flanked by Tol2 elements (Kawakami et al., 2004), were injected into zebrafish 
eggs at the single cell stage. Transposase RNA was prepared using the Ambion mMessage mMachine SP6 kit (http://www.thermofisher.com, RRID: SCR_008406). Tol2-GFP plasmid and transposase were diluted to a final concentration of $50 \mathrm{ng} / \mu \mathrm{l}$. Injection into fertilized zebrafish eggs was performed as previously described (Ono et al., 2001). Surviving larvae were raised to adulthood. Nervous systems of embryos obtained from outcrosses were screened for fluorescence, and stable lines (at least three generations of outcrosses) were developed. GE4a, with GFP observed in both pupil and hindbrain, was identified as a line of interest for retinal studies. The transgene was located by inverse polymerase chain reaction (PCR), as previously described (Ikenaga et al., 2011).

To identify GFP-labeled retinal neurons in GE4a, live in vitro flattened adult eyecups (Connaughton and Nelson, 2010) or live adult retinal sections (Connaughton, 2003) were prepared from outcrossed adults. The tissue was maintained in Leibovitz L15 medium (Invitrogen, https://www.thermofisher.com), and observed by confocal microscopy (Zeiss 510 Meta, http://www.zeiss.com/microscopy). Z-axis projections of image stacks (two to four planes separated by $3 \mu \mathrm{m}$; ZEN software, http://www.zeiss.com/microscopy, RRID: SCR_013672), together with differential interference contrasting (DIC) imaging of retinal layers, served to identify cell body and dendritic layering patterns.

\section{Preparation and perfusion of zebrafish retina eyecups}

Light-adapted adult zebrafish (Danio rerio) were decapitated with a fresh single-edged razor. Eyes were removed, carefully cleaned of attached tissue, and mounted upright on 5-10-mm squares of black nitrocellulose filter paper (cat\# HABP02500, Millipore, http:// www.emdmillipore.com). Under a binocular microscope (MZ12-5, Leica, http://www.leicamicrosystems.com, RRID: SCR_008960), the anterior segment and lens were removed. Short radial cuts were made in the limbus, allowing the eyecup to flatten for microelectrode and imaging access.

The eyecup, adhering to nitrocellusose paper, was submerged in the recording chamber and superfused $(0.3 \mathrm{ml} / \mathrm{min})$ from the vitreal side with a 28 -gauge MicroFil syringe needle (WPI, http://www.wpiinc.com, RRID: SCR_008593). The perfusate was minimum essential medium (MEM; glutamine-, HEPES-, and pyruvate-free, Invitrogen, cat\# 11090-099, https://www.thermofisher.com). Prior to infusion, MEM was equilibrated by bubbling with 95\% $\mathrm{O}_{2} / 5 \% \mathrm{CO}_{2}$. MEM is an Earle's salts, bicarbonate-buffered, cell culture medium containing amino acid and vitamin supplementation. All recordings were performed at room temperature $\left(22^{\circ} \mathrm{C}\right)$. Stable electroretinographic (ERG) b-wave responses are recorded from zebrafish eyecups for periods in excess of 10 hours using this medium (Nelson and Singla, 2009). Extra eyecups were maintained in bubbled MEM for later use the same day.

The recording chamber, mounted beneath the objective of a fixed-stage Olympus BX51 wide-field microscope (Olympus America, http://www.olympuslifescience.com), served for stimulation, perfusion, and imaging. A $4 \times$ air objective was used for electrode placement and for light stimulation. 


\section{Electrodes, recording, and staining}

The tips of sharp microelectrodes, pulled to a shank length of $\sim 15 \mathrm{~mm}$ using a Flaming/ Brown puller (model P-87, Sutter Instruments, http://www.sutter.com), were filled with Alexa Fluor 594 or 488 hydrazide in 0.2M KCL (Molecular Probes, cat\# A10442, cat\# A10436, https://www.thermofisher.com, RRID: SCR_013318). The remainder of the electrode was filled with 0.3M KCL. Mean input resistance (while intracellular) was 724 $\pm 514 \mathrm{M}(n=37)$. Cells were penetrated in the dark, without visualization. The mean z-axis depth of encounter relative to electrode touch on the retinal surface was $47 \pm 27 \mu \mathrm{m}(n=32)$. Penetration was signaled by the appearance of responses to the test stimuli $(570 \mathrm{~nm}, 600$ msec duration, 3-s interval), and was often accompanied by a negative-going "penetration" or membrane potential $(-21 \pm 11 \mathrm{mV}$, mean $\pm \mathrm{SD}, n=35$; example in Fig. 1A). These potential changes on penetration were similar in amplitude to those reported in amacrine cells of other species: -30 to $-40 \mathrm{mV}$ in Necturus (Werblin and Dowling, 1969) and -25 $\mathrm{mV}$ in cat (Nelson, 1982). Maximum onset response amplitudes averaged $2.4 \pm 2.2 \mathrm{mV}$ (mean $\pm \mathrm{SD}, n=36$ ). These microelectrode voltage signals were amplified with an Axopatch 200B amplifier (Molecular Devices, http://www.moleculardevices.com) in current clamp mode, and digitized (0.5-msec sample rate) with a Digi-Data 1440A and pCLAMP 10 software (http://www.moleculardevices.com, RRID: SCR_011323). Alexa dyes were injected electrophoretically (+300 nA, 15 seconds). After electrophoresis of dye from the microelectrode, the tip was seen within the cell body or major dendrite of a stained amacrine cell, with the cell body at the distal edge of the IPL. All recordings were made during the 14-hour day cycle of the zebrafish habitat, using a dark recording cage without imposed background illumination. Traces were imported, displayed, and analyzed in Origin software using in-house Labtalk scripts (v. 8 to v. 2016, OriginLab, http://www.Origin-Lab.com). The root mean square (RMS) signal amplitude for an interval either during or after stimulation (Fig. 1A) was the response index used for spectral model fits. Unlike peak measurements, which can occur at different time points (Fig. 1A), the RMS interval is fixed in time. For most waveforms the interval was either the first $300 \mathrm{msec}$ of stimulation or the $300 \mathrm{msec}$ following stimulation. These time segments isolate and encompass onset and offset transients (Fig. 1A), which peak at $\sim 100 \mathrm{msec}$. For some waveforms the interval was adjusted to isolate a component of different time course. In the RMS measure, a baseline is determined from the mean membrane potential $100 \mathrm{msec}$ preceding stimulation. In the example of the 300-msec measurement interval, the mean baseline is subtracted from each of 600 voltage measurements made within the interval; the difference is squared, summed, and divided by 600 , and the square root is taken. A linear fit of RMS amplitude on troughto-peak amplitude provides the scaling factor to convert the RMS value to "scaled RMS." The correspondence of "scaled RMS" and "trough-to-peak" amplitudes appears in Figure 1B. The use of many points in computing RMS amplitude integrates a full response component and improves the signal-to-noise ratio. Although applicable to complex waveforms with multiple peaks, RMS measures are here applied only to single peaked response elements. Positive and negative RMS amplitudes were distinguished by the mean amplitude during the response interval. 


\section{Optical source and calibration}

Stimuli were delivered through the microscope epifluorescence port using a $4 \times$ UV Plan Apo objective. A xenon source, focused onto a liquid light guide through intervening filter wheels (Lambda 10-2, Sutter Instruments, http://www.sutter.com), provided the stimulus light. Interference filters selected stimulus wavelengths from 330 to $650 \mathrm{~nm}$ at $40-\mathrm{nm}$ intervals (20-nm halfband, Chroma Technology, https://www.chroma.com). Metallic ND filters provided stimulus attenuations from 0.5 to $7.5 \log$ units (Andover, https:// www.andovercorp.com). Monochromatic illumination was measured as quantal flux using a calibrated silicon detector (Newport, http://www.newport.com). Maximum intensities ranged from $6.3 \log \left(h v \cdot \mu \mathrm{m}^{-2} \cdot \mathrm{s}^{-1}\right)$ at $330 \mathrm{~nm}$ to $8.0 \log \left(h v \cdot \mu \mathrm{m}^{-2} \cdot \mathrm{s}^{-1}\right)$ at $490 \mathrm{~nm}$, where $h v$ is the symbol for quanta. To study amacrine cell spectral responses, four different stimulus protocols, fixed sequences of wavelength and brightness, were computer generated. These protocols contained 9-27 broad field stimuli with wavelengths ranging from $650 \mathrm{~nm}$ (red) to $330 \mathrm{~nm}$ (UV), and irradiances ranging from 0.5 to $7 \log \left(h v \cdot \mu \mathrm{m}^{-2} \cdot \mathrm{s}^{-1}\right)$. For slit stimulation an aperture was moved through the epifluorescence back focal plane using an SMC100CC linear actuator (Newport, http://www.newport.com).

\section{Cone-signal analysis}

We modeled amacrine-cell light responses to result from a linear sum of one to four cone signals. Each cone signal is represented as an independently saturable Hill function (Nelson and Singla, 2009). The model is not based on synaptic pathways for individual cone inputs but says simply that, whatever the pathway used, the signals arising from cones with different opsin types are separately saturable. The model has previously been used to characterize the spectral responses of zebrafish horizontal cells (Connaughton and Nelson, 2010), and of the zebrafish electroretinogram (Nelson and Singla, 2009). This cone-signal extraction model is given by:

$$
V=\frac{\mathrm{V}_{\mathrm{r} 570} I}{I+\mathrm{k} / \mathrm{A}_{\mathrm{r} 570}(\lambda)}+\frac{\mathrm{V}_{\mathrm{g} 480} I}{I+\mathrm{k} / \mathrm{A}_{\mathrm{g} 480}(\lambda)}+\frac{\mathrm{V}_{\mathrm{b} 415} I}{I+\mathrm{k} / \mathrm{A}_{\mathrm{b} 415}(\lambda)}+\frac{\mathrm{V}_{\mathrm{u} 362} I}{I+\mathrm{k} / \mathrm{A}_{\mathrm{u} 362}(\lambda)}
$$

The RMS amacrine-cell light response amplitudes ( $V$ ) for stimuli of different wavelengths $(\lambda)$ and irradiances $(I)$ were fit by this three-dimensional (3D), five-parameter nonlinear least squares analysis (Eq. 1). Four of the fit parameters are Vr570, Vg480, Vb415, and Vu362, the saturation voltages for red, green, blue, and UV cone signals, respectively. A single half-saturation irradiance at peak opsin absorbance (k) is applied to all cones. For offpeak wavelengths, $k$ is divided by the peak-normalized opsin absorbance (A) for the cone type at the stimulus wavelength $(\lambda)$. Given the larger datasets obtained in ERG recordings, better spectral fits were achieved by allowing a separate k-value for each cone type (Nelson and Singla, 2009). For the typically more limited datasets available in single cell physiology, a single k-value is applied to all cones, and the amount of signal contribution from each cone is characterized only by the saturation voltage $\mathrm{V}$.

We used the microspectrophotometry/suction-electrode-based absorbance peaks provided by Robinson et al. (1993) and Palacios et al. (1996). These are red/LWS1 (570 nm), 
green/RH2 $(480 \mathrm{~nm})$, blue/SWS2 $(415 \mathrm{~nm})$, and UV/SWS1 $(362 \mathrm{~nm})$. More recent patchelectrode spectra from adult zebrafish cones provide wavelength maxima substantially in agreement (Endeman et al., 2013). Collectively, zebrafish cones express eight opsins, and additionally combine with both vitamin A1 and A2 chromophores (Chinen et al., 2003; Endeman et al., 2013). For this reason, the modeled cone spectral peaks are based on microspectrophotometery/electrophysiology of cone types rather than on exogenously expressed opsin absorbance peaks.

$\operatorname{Ar} 570(\lambda), \operatorname{Ag} 480(\lambda), \operatorname{Ab} 415(\lambda)$, and $\operatorname{Au362}(\lambda)$ (Fig. 1C) are the normalized absorbance functions for red, green, blue and UV cone opsins, respectively. These functions are calculated from 8th order polynomial-based nomograms. For red, green, and blue cones, the Hughes et al. (1998) nomogram is used. For the UV cone, the Palacios et al. (1996) nomogram is used. Nomogram polynomial coefficients are summarized in Nelson and Singla (2009). The secondary $366-\mathrm{nm}$ peak in the $570-\mathrm{nm}$ (red cone) template is the $\beta$-band. In neurons that mix signals from different cone types, UV cones might also contribute to peaking in this region (Fig. 1C), as might blue cones. Equation 1 provides a rational scheme for finding the most likely signal combination.

\section{Statistical Analysis}

The criteria for accepting a microelectrode recording were: 1) a stained amacrine cell at the microelectrode tip, with dendrites traceable in Neurolucida; 2) a signal-to-noise ratio sufficient to measure amplitudes of the light response waveforms; 3) sufficient response stability for spectral sensitivity analysis; and 4) identification of significant spectral components. Repeats of intensity-response series at $650 \mathrm{~nm}$ served as one measure of stability, and curve-fit parameter $t$-tests were a measure of component significance. The minimum stimulus number was nine. This nine-stimulus protocol presented all wavelengths with a single fixed irradiance, and was the sequence used prior to dye injection. Four other stimulus protocols ranged from 20 to 27 stimuli. The largest modeled sequence was 80 consecutive responses. To achieve this number, multiple stimulus protocols were delivered, so that responses to a greater range of wavelengths and irradiances could be modeled.

Cone signals (Vr570, Vg480, Vb415, and Vu362) were extracted from responses to stimulus protocols using 3D nonlinear least squares fits (NLSM, Origin, http://www.OriginLab.com) to Equation 1. The fits provide both the amplitude and standard error (SE) for these saturation voltages. A two-tailed $t$-test (Prism v. 6, GraphPad Software, www.graphpad.com, RRID: SCR_002798) was used to determine whether any of these saturation voltages differed from 0 (null hypothesis, $a<0.05$ ). The $t$-value for this test is the saturation voltage divided by its SE, with the degrees of freedom being $n-5$ ( $n$ is the number of stimuli, and the model has five fit parameters). Because $\mathrm{V}>0$ indicates depolarizing signals, and $\mathrm{V}<0$ indicates hyperpolarizing signals, we allow $t$ to be positive or negative.

Best fit spectral curves are interpolated using the five fit parameters (Eq. 1) These provide amplitude responses for a hypothetical stimulus of constant irradiance across all wavelengths. The brightness of this stimulus affects shape. If too bright, a saturating response is calculated at all wavelengths. If too dim, an extrapolation into stimulus regions not actually used occurs. For most curves, a constant quanta irradiance of $2.5 \log \left(h \nu \cdot \mu \mathrm{m}^{-2}\right.$.s 
${ }^{-1}$ ) was used, $0.3 \log$ units below the mean half-saturation value for amacrine cells in this group of stains. The mean $\log (\mathrm{k})$ value for amacrine cells in this study was $2.8 \pm 0.6$ ( $\mathrm{SD}, n$ $=35$ ), or about $560 h v \cdot \mu \mathrm{m}^{-2} \cdot \mathrm{s}^{-1}$. An example of a semisaturating ON response to a $570-\mathrm{nm}$ stimulus at $2.0 \log \left(h v \cdot \mu \mathrm{m}^{-2} \cdot \mathrm{s}^{-} 1\right), 100 h v \cdot \mu \mathrm{m}^{-2} \cdot \mathrm{s}^{-1}$, appears in Figure 1A (570 6.0). The mean $\log (\mathrm{k})$ values for $\mathrm{L} 2$ monophasic horizontal cells in a companion study (Connaughton and Nelson, 2010) was $3.4 \pm 0.5$ (SD, $n=86)$. Zebrafish amacrine cells appear significantly more sensitive than horizontal cells $(P<0.0001, t$-test $)$.

\section{Collection of image stacks and 3D reconstruction}

After recording light responses and electrophoresing Alexa Fluor dyes, z-axis image stacks of stained neurons were obtained using a $40 \times$ LUMPlanFL/IR lens (numerical aperture 0.8 water, Olympus America, http://www.olympus-lifescience.com), a Retiga 2000RV cooled CCD camera (Qimaging, http://www.qimaging.com), a z-axis controller (Prior Optiscan, http://www.prior-us.com), epifluorescence filter sets (Texas red or eGFP, Olympus America), and Metamorph imaging software (Molecular Devices, http:// www.moleculardevices.com, RRID: SCR_002368). The Retiga 2000RV camera with $40 \times$ objective captured a field of $250 \mu \mathrm{m}$ width at an xy-axis resolution of $0.2 \mu \mathrm{m}$ per pixel. The z-axis resolution was set in Metamorph at $0.6 \mu \mathrm{m}$ per image. Each stack contained 100-200 images. The acquisition time was $\sim 5$ minutes. For some stains, up to six stacks were required to cover the field. Image acquisition was limited by Alexa Fluor bleaching.

Color look-up tables were added to image stacks using NIH ImageJ (http:// rsb.info.nih.gov/ij, RRID: SCR_003070). For Alexa 594 microelectrode stains on the GE4a transgenic background, image stacks with appropriate color look-up tables were combined in ImageJ, creating a two-color image stack. Finally, ImageJ created a TIFF file from the Metamorph image stacks that could be read by Neurolucida (MBF Bioscience, http:// www.mbfbioscience.com, RRID: SCR_001775). After completing en face 3D drawings of microelectrode stains, the reconstruction was rotated to reveal amacrine dendritic branching patterns within the IPL. To quantify branching strata, IPL boundaries were determined from the z-axis locations of transgenic amacrine and ganglion cells within the same image stack, or in the case of wild-type retinas, from the injected cell body position and from knowledge of IPL thickness gained from the GE4a transgenic. The stratification depth was measured by examining image stacks in ImageJ. The predominant pattern of amacrine dendrites is planar branching from a central stalk (Connaughton et al., 2004; Yeo et al., 2009). For such branching, stratification level can be determined at any point in the dendritic field, although most typically, stratification depth was measured close to the injected cell body. Dendritic areas were estimated from major and minor axes using the elliptical approximation ( $\pi \mathrm{ab})$.

\section{RESULTS}

\section{Determining IPL boundaries from GFP-labeled amacrine and ganglion cells}

The GE4a transgenic was used as a background stain to locate IPL strata in flat-mount view. In GE4a larvae, hindbrain, heart, and eye fluoresce (Fig. 2A). Labeled retinal neurons can be seen through the pupil (Fig. 2A). Inverse PCR from GE4a larvae revealed the site of transgene insertion in a noncoding region of chromosome 14 (Chr14:42,668,063- 
$42,668,401)$. The location is about $100 \mathrm{~kb}$ downstream of dystrophin-related protein 2 (DRP2). When adult GE4a retinas are isolated and sliced (Connaughton, 2003), select horizontal-cell, amacrine-cell, and ganglion-cell populations fluoresce (Nelson et al., 2009), together with GFP-labeled processes within the IPL (Fig. 2B).

Six IPL strata (s1-s6; Fig. 1B) provide a basis for distinguishing amacrine-cell and ganglion-cell dendritic branching patterns, and for understanding IPL functional layering. In GE4a, not all amacrine cells express GFP, and the marked amacrine cells are of more than one type. Amacrine cells with dendrites restricted to either the ON (s4-s6) or OFF (s1-s3) IPL sublaminae are shown in Figure 2B (Aon, Aoff), along with a single type of GFPexpressing ganglion cell (Gon). Ganglion-cell labeling is best appreciated en face in the flattened eyecup preparation (Fig. 2C). While the density of amacrine labeling is constant across the retina, labeled ganglion cells are densest near the optic disk, and become sparser in the periphery. In Figure 2C, axons of labeled ganglion cells are seen to travel through the optic fiber layer curving toward the optic disk, just beyond the upper right corner of the image.

In GE4a the inner and outer boundaries of the IPL are flanked by fluorescent amacrine and ganglion cell bodies. Using these cell body markers, IPL thickness in live adult eyecups was $40.7 \pm 4.0 \mu \mathrm{m}(n=13$, mean $\pm \mathrm{SD})$; correspondingly, each of the 6 IPL strata (s1-s6) was $6.8 \mu \mathrm{m}$ in thickness. In GE4a, the IPL depth of dendritic branching for any amacrine dendrite can be determined by reference to the depths of GFP-labeled cell bodies. In Figure 2D the dendrites of an amacrine cell stained through the microelectrode with Alexa 594 hydrazide are seen to course among GFP-labeled amacrine cell bodies in an OFF-type branching pattern. The constancy of IPL thickness in zebrafish meant that branching levels for amacrine cells stained in wild-type zebrafish could also be determined, as it was only necessary to know the depth of the microelectrode-stained amacrine cell body to determine the stratum occupied by an amacrine dendritic branch point or process beneath it.

\section{ON-OFF physiology in $a / b$ bistratified amacrine cells}

There are two major response components for ON-OFF amacrine cells: transient depolarization following stimulus onset, and transient depolarization following stimulus offset. In some cells, there is a third component: a lower amplitude, sustained depolarization throughout the stimulus, particularly with brighter stimuli (Fig. 1A). The latter are termed ON-OFF-Sustained. Of 21 ON-OFF or ON-OFF-Sustained cells (Table 1), 14 were $a / b$ bistratified. In the example of Figure 3, microelectrode recording revealed transient depolarization at both light onset and offset, an ON-OFF response. All test wavelengths (330-650 nm) evoked ON and OFF components (Fig. 3A). Onset and offset transients for the 27-stimulus trial were fit to the spectral model (Eq. 1), providing constant-quanta amplitude spectra for each response component (Fig. 3B). Amplitudes peaked at $567 \mathrm{~nm}$ for the ON component and at $566 \mathrm{~nm}$ for the OFF component. The ON component contains only red (Ar570) cone signals. The red cone saturation voltage at $\mathrm{ON}$ is depolarizing and highly significant $(\operatorname{Vr} 570 \neq 0: t=19.7, P<0.0001, n=27)$, while the signals from green cones (Vg480 $\neq 0: t=0.7, P=0.658, n=27)$, blue cones (Vb415 $\neq 0: t=-1.6, P=0.121, n$ $=27$ ), and UV cones (Vu362 $\neq 0: t=-0.6, P=0.529, n=27$ ) were not significantly different 
from 0. Like the $\mathrm{ON}$ transient, the OFF transient was composed only of red cone signals $(\mathrm{Vr} 570 \neq 0: t=10.2, P<0.0001, n=27)$. No other cone signal was detected $(\mathrm{Vg} 480 \neq 0: t=$ $1.0, P=0.325$; Vb415 $\neq 0: t=0.2, P=0.873$; Vu362 $\neq 0: t=-.3, P=0.773 ; n=27)$.

After injecting this neuron with Alexa 594 hydrazide, the dendritic branching pattern was traced from widefield image stacks in Neurolucida, revealing a dendritic field of $182 \times 120$ $\mu \mathrm{m}$. In flat-mount view, the branching is dense, and many dendrites cross, consistent with several planes of branching (Fig. 3C). Rotation of the reconstruction (Fig. 3D) reveals a bistratified branching pattern in s2 and s5 of the IPL. Individual dendrites jump from one layer to the other, giving the z-axis pattern a laddered appearance.

In another example of an $a / b$ bistratified amacrine cell (Fig. 4), depolarizing voltage transients occur both at light onset and offset for all test wavelengths (Fig. 4A). Onset and offset amplitudes to the 38-stimulus trial were fit to the spectral model (Eq. 1), providing constant-quanta amplitude spectra (Fig. 4B). Spectral peaks for both ON and OFF transients were found at $566 \mathrm{~nm}$. Red cone depolarization was highly significant both at ON (Vr570 $\neq$ 0: $t=29.8, P<0.0001, n=38$ ) and at OFF (Vr570 $\neq 0: t=15.9, P<0.0001, n=38)$. No significant signals from other cone types were found in either the $\mathrm{ON}$ or the OFF components $(\mathrm{ON}: \mathrm{Vg} 480 \neq 0, t=0.9, P=0.369$; Vb415 $\neq 0, t=0.2, P=0.833$; Vu362 $\neq 0, t$ $=-0.1, P=0.898$; OFF: Vg480 $\neq 0, t=1.3, P=0.190 ; \mathrm{Vb} 415 \neq 0, t=0.6, P=0.556 ; \mathrm{Vu} 362$ $\neq 0, t=0.4, P=0.668 ; n=38)$.

The dendritic branching pattern, traced in Neurolucida, revealed a dendritic field $143 \times 100$ $\mu \mathrm{m}$ in diameter, with an axon $183 \mu \mathrm{m}$ in length (Fig. 4C). In flatmount view, densely branched, varicose, crossing dendrites are seen. Rotation of the reconstruction (Fig. 4D) reveals a bistratified branching pattern in s2 and s4 of the IPL. The axon remains in the s2 plane.

\section{ON-OFF physiology for amacrine cells stratifying in IPL sublamina a}

In 7 of $21 \mathrm{ON}-\mathrm{OFF}$ or ON-OFF-Sustained amacrine cells, dendritic stratification was restricted to sublamina $a$ (Table 1). Branching patterns in these cells could be either monostratified $(n=3)$ or bistratified $(n=4)$. No axon-like processes were seen in any of these neurons.

In the unit of Figure 5A transient depolarizations at stimulus onset and offset occur for all wavelengths. In addition, a maintained depolarization is present throughout the stimulus. This three-component waveform is termed "ON-OFF-Sustained." Onset and offset transients from the 48-stimulus trial were fit to the spectral model (Eq. 1). The onset peak sensitivity occurred at $567 \mathrm{~nm}$, and the offset peak at $568 \mathrm{~nm}$ (Fig. 5B). In both components red cone depolarization was the most significant (ON: Vr570 $\neq 0, t=24.8, P<0.0001$; OFF: $\mathrm{Vr570} \neq 0, t=24.7, P<0.0001 ; n=48)$. The onset transient was depolarized significantly, but to a lesser extent ( $30 \%$ of Vr570) by blue cone signals (Vb415 $\neq 0: t=2.9, P<0.01, n$ $=48)$. Green cone signals (ON: Vg480 $\neq 0: t=1.1, P=0.277$; OFF: $\operatorname{Vg} 480 \neq 0, t=0.0, P=$ 0.999; $n=48$ ) and UV cone signals (ON: Vu362 $\neq 0: t=0.7, P=0.485$; OFF: Vu362 $\neq 0, t=$ $0.0, P=0.997 ; n=48$ ) did not contribute to either transient (Fig. 5B). No blue cone signal was evident in the OFF transient (Vb415 $\neq 0, t=0.756, P=0.521, n=48$ ). 
Dendritic reconstruction reveals a semilunate field structure (Fig. 5C). The dendritic extent is large, $418 \times 147 \mu \mathrm{m}$. Crossing dendrites occur in the central portion of the field. The $\mathrm{z}-$ axis projection (Fig. 5D) reveals a tightly bistratified pattern in IPL $s 1$ and $s 3$. The process appearing to project above the s1 plane results from a dendritic projection climbing a tissue fold.

In the unit illustrated in Figure 6A, transient depolarizations at stimulus onset and offset again occur for all wavelengths. Onset responses for the 60-stimulus trial were fit to the spectral model (Eq. 1). A major spectral peak occurs at $569 \mathrm{~nm}$ for the ON transient, and at $568 \mathrm{~nm}$ for the OFF transient. Red cone signals are depolarizing for both transients (ON: Vr570 $\neq 0, t=21.9, P<0.0001$; OFF: Vr570 $\neq 0, t=7.9, P<0.0001 ; n=60)$. Low-level hyperpolarizing influences were detected in the onset response from both green $(\sim 15 \%$ of Vr570) and UV ( 20\% of Vr570) cone signals (Vg480 $\neq 0, t=-2.0, P=0.05$; Vu362 $\neq 0, t$ $=2.3, P<0.05 ; n=60)$, but no other cone signals appeared to influence either the $\mathrm{ON}$ or OFF components (ON: Vb415 $\neq 0, t=1.6, P=0.108$; OFF: Vg480 $\neq 0, t=0.2, P=0.838$; Vb415 $\neq 0: t=0.7, P=0.491 ; \mathrm{Vu} 362 \neq 0: t=0.5, P=0.598 ; n=60)$. Dendritic reconstruction reveals an elliptical field structure (Fig. 6C). The dendritic extent is small, $147 \times 92 \mu \mathrm{m}$, and there are no crossing dendrites. The z-axis rotation (Fig. $6 \mathrm{D}$ ) reveals a narrowly monostratified pattern in IPL $s 3$.

\section{Amacrine cells with OFF physiology}

The OFF waveforms of zebrafish amacrine cells were either 1) temporally two-component, with a maintained light-induced hyperpolarization followed by transient OFF depolarization ( $n=2$, Table 1$)$; or 2 ) temporally three-component, characterized by three transients. The first is onset hyperpolarization, the second is delayed onset depolarization, and the third is offset depolarization ( $n=2$; Table 1). The dendrites of amacrine neurons with either of these OFF physiologies were monostratified in s1 or s2 of IPL sublamina $a$.

A two-component OFF amacrine physiology is shown in Figure 7A. This cell was spontaneously active, with impulse frequency modulated by the membrane potential. For wavelengths from 330 to $650 \mathrm{~nm}$, a sustained hyperpolarization inhibits spiking during the light stimulus (component 1), while transient depolarization at stimulus offset triggers impulse bursts (component 2). To evaluate spectral properties (Fig. 7B), impulse activity was filtered out so that the amplitude of ON and OFF components could be modeled (Eq. 1). The onset hyperpolarization peaked at $572 \mathrm{~nm}$, and the offset depolarization peaked at $568 \mathrm{~nm}$. Red cone signals dominate both ON hyperpolarization (Vr570 $\neq 0$ : $t=-18.4, P<0.0001 ; n$ $=51)$ and OFF depolarization $(\operatorname{Vr} 570 \neq 0, t=19.2, P<0.0001 ; n=51)$. No other cone signal was significant for ON hyperpolarization (Vg480: $t=0.2, P=0.838$; Vb415: $t=1.2$, $P=0.234 ;$ Vu362: $t=1.0, P=0.342 ; n=51)$. While a subtle green cone hyperpolarizing influence ( 20\% of Vr570) is inferred for the OFF transient, signals from blue or UV cones were not significant (Vg480: $t=2.1, P<0.05$; Vb415: $t=0.3, P=0.792$; Vu362: $t=0.3, P=$ 0.739). The dendritic extent of the Alexa 594 stain (Fig. 2D) was reconstructed (Fig. 7C), revealing a large elliptical field $(319 \times 150 \mu \mathrm{m})$, eccentric cell body, dense branching, and crossed dendrites. The z-axis rotation (Fig. 7D) shows a thick, monostratified pattern centered in IPL s2. 
A temporally three-component OFF-amacrine is shown in Figure 8A. Transient hyperpolarization at stimulus onset (component 1 ) is followed by a later developing $\mathrm{ON}$ depolarization (component 2). At stimulus offset there is transient depolarization (component 3). The spectrum of onset hyperpolarization differs from offset depolarization (Fig. 8B). Onset hyperpolarization peaks at $431 \mathrm{~nm}$; offset depolarization, at $560 \mathrm{~nm}$. In the onset hyperpolarization significant signals arise from red and blue cones $(\mathrm{Vr} 570 \neq 0: t=$ $-3.1, P<0.005 ; \mathrm{Vb} 415 \neq 0: t=-3.2, P<0.005 ; n=36$ ). In the OFF depolarizing transient only red cone signals are significant ( $\operatorname{Vr} 570 \neq 0: t=6.1, P<0.0001, n=36$ ). It is difficult to develop an index that separates delayed depolarization from onset hyperpolarization. The analysis of the trough-to-peak amplitude for the delayed depolarization is qualitatively similar to the onset hyperpolarization, with significant signals from both red and blue cones (Vr570 $\neq 0, t=2.7, P<0.05 ; \mathrm{Vb} 415 \neq 0, t=3.1, P<0.005 ; n=36$ ). Dendritic reconstruction (Fig. 8C) reveals an elliptical field $(200 \times 114 \mu \mathrm{m})$ with an eccentric cell body. Except for the longest process, possibly an axon, the dendrites are uncrossed. The zaxis rotation (Fig. 8D) reveals a tightly monostratified pattern in IPL s1.

\section{Properties of ON and OFF depolarizing transients}

Amacrine depolarizing transients from non-opponent cells are all spectrally similar. The ON and the OFF transients in ON-OFF cells (Figs. 3B-6B) and the OFF transients in OFF cells (Figs. 7B, 8B) are dominated by signals from red cones. In Figure 9A, the mean spectral sensitivities of ON and OFF responses from ON-OFF cells are compared with the Ar570 red cone absorbance template (Hughes et al., 1998). Onset spectra, offset spectra, and the red cone template curves all peak together. The transient OFF depolarizations of OFF amacrine cells are also well fit by the red cone template (Fig. 9B), even though the onset component of these same cells can be spectrally mixed.

Peak times for amacrine depolarizing transients are similar. These are derived from the mean waveform within the dataset for each cell. Use of the cellular mean response reduces the influence of noise fluctuations on peak measurements, and gives an average over stimulus wavelength and brightness. For ON-OFF amacrine responses, the mean time from stimulus onset to the depolarizing peak in the mean waveform was $79 \pm 3 \mathrm{msec}$ (mean $\pm \mathrm{SE}, n=20$ cells). For the OFF depolarization the peak time was $105 \pm 6 \mathrm{msec}(\mathrm{mean} \pm \mathrm{SE}, n=20)$. For OFF amacrine responses, the peak time for OFF depolarization was $108 \pm 24$ msec (mean \pm $\mathrm{SE}, n=4)$.

In seven ON-OFF cells, receptive fields were mapped with slits. These cells included both $a / b$ bistratified types, and types branching only in sublamina $a$. ON-OFF responses were evoked throughout the receptive field in all but one of these cells. The lone exception, a sublamina a monostratified type, was ON-OFF in the center, and OFF in the surround. An example of a homogeneous ON-OFF receptive field appears in Figure 9C. ON-OFF waveforms are evoked by all slit positions. Only the amplitudes diminish in the peripheral field. 


\section{Amacrine cells with sustained ON physiology}

Another waveform found among amacrine light responses was a sustained depolarization ( $n$ = 7; Table 1). A depolarizing transient often preceded the sustained component, but there was no matching depolarizing transient at stimulus offset, unlike ON-OFF responses. The dendritic arbors of these neurons were monostratified in s4 of IPL sublamina $b$ (six of seven cases; Table 1). The remaining example was also monostratified, in nearby s3 of IPL sublamina $a$.

In the example of Figure 10A, depolarization was sustained throughout light stimulation, regardless of stimulus wavelength. Transient hyperpolarization occurred at stimulus offset. Constant-quanta amplitude spectra at stimulus onset (Fig. 10B) revealed a midspectral peak at $537 \mathrm{~nm}$. The contributing cone signals originate with a combination of red (Ar570) and green (Ag480) cone input signals. Both red and green cone saturation voltages are significant (Vr570 $\neq 0: t=15.4, P<0.0001 ; \mathrm{Vg} 480 \neq 0: t=3.2, P<0.01 ; n=18$ ). Blue and $\mathrm{UV}$ cone signals were not significant contributors to this response (Vb415 $\neq 0: t=-.4, P=$ 0.663 ; Vu362 $\neq 0: t=0.4, P=0.668 ; n=18$ ). Dendritic reconstruction (Fig. 10C) reveals a large round, almost "starburst" field $(316 \times 320 \mu \mathrm{m})$ with a central cell body. The dendrites are spiny and do not cross. No axon is evident. The z-axis rotation (Fig. 10D) reveals a narrowly monostratified pattern in s4, IPL sublamina $b$.

\section{Amacrine cells with color-opponent physiology}

Some zebrafish amacrine cells were color-opponent or "C-type" $(n=4$; Table 1$)$. The onset responses of C-type neurons are depolarizing at some wavelengths, but hyperpolarizing at others. Similar to zebrafish horizontal cells (Connaughton and Nelson, 2010) both spectrally biphasic and spectrally triphasic physiologies were encountered, but the patterns of wavelength depolarization and hyperpolarization differed from C-type horizontal cells. The dendrites of C-type amacrine cells, regardless of wavelength patterns, were monostratified in IPL sublamina $b$ (four of four; Table 1), with three of four in s5 (Table 1).

A UV-depolarized, spectrally biphasic C-type amacrine cell is illustrated in Figure 11. For short wavelengths (330 and $410 \mathrm{~nm}$ ), stimulation depolarizes the membrane in sustained fashion. For longer wavelengths (490, 570, and $650 \mathrm{~nm})$, hyperpolarization prevails; this is blue-yellow opponency (Fig. 11A). Because midspectral stimulation evoked a pattern of transient depolarization together with sustained hyperpolarization, the transient $(0-100$ msec) and sustained (100-600 msec) components were treated as separate processes (Fig. 11B). Both transient and sustained components are spectrally biphasic. In the analysis of each time segment (Eq. 1, Fig. 11B) both blue and UV depolarizing signals were significant (transient: Vu362 $\neq 0, t=6.3$; Vb415 $\neq 0, t=5.7$; both $P<0.0001$; sustained: Vu362 $\neq 0, t=$ 8.4; Vb415 $\neq 0, t=7.8$; both $P<0.0001 ; n=71)$. In each time segment red hyperpolarization was significant (transient: $\operatorname{Vr} 570 \neq 0, t=-3.9$; sustained: $\operatorname{Vr} 570 \neq 0, t=$ -3.0 ; both $P<0.005 ; n=71$ ). In the sustained component an additional green cone hyperpolarization was evident $(\mathrm{Vg} 480 \neq 0, t=-3.5, P<0.005, n=71)$. This later-occurring additional green signal shifted the hyperpolarization peak from 562 to $506 \mathrm{~nm}$ (Fig. 11B). Considering both transient and sustained time segments, red, green, blue, and UV signals all contribute to the biphasic spectrum. Dendritic reconstruction (Fig. 11C) reveals a large, 
somewhat asymmetric, loosely branched arbor with sparse dendritic crossing $(319 \times 200$ $\mu \mathrm{m}$; the rectangular shape suggests that dendritic tips were too dim to reconstruct). No axon is evident. The z-axis rotation (Fig. 11D) reveals a monostratified pattern in IPL s5.

A spectrally triphasic C-type amacrine signal appears in Figure 12. This cell is depolarized by UV stimulation (Fig. 12A, $370 \mathrm{~nm}$ ), but with blue stimuli (Fig. 12A, $410 \mathrm{~nm}$ ) the depolarization is erased, reappearing with midspectral $(490 \mathrm{~nm})$ stimuli. The spectral analysis is broken into two time segments: transient (0-100 msec) and sustained (100-600 msec). Constant-quanta amplitude spectra (Eq. 1) are generated for each segment (Fig. 12B). The transient is nearly monophasic in spectral shape, with a single peak at $362 \mathrm{~nm}$. For the onset transient, UV depolarization is the only significant signal (Vu362 $\neq 0: t=16.4, P<$ $0.0001, n=33$ ). In the sustained segment, UV depolarization is also significant (Vu362 $\neq 0$ : $t=5.3, P<0.0001, n=33$ ). In addition, blue hyperpolarization is significant (Vb415 $\neq 0: t=$ 3.2, $P<0.005, n=33$ ), and green depolarization is significant ( $\mathrm{Vg} 480 \neq 0: t=4.7, P<$ $0.0001, n=33$ ). Opposing signals arising from green, blue, and UV opsins combine in a spectrally triphasic fashion, with depolarizing peaks at 358 and $484 \mathrm{~nm}$, and a hyperpolarizing trough at $418 \mathrm{~nm}$. Examination of responses to red stimulation (Fig. 12A, 570 and $650 \mathrm{~nm}$ ) suggest an additional red cone hyperpolarization, which would make the cell spectrally tetraphasic. In neither time segment was the $\mathrm{Vr} 570$ signal strong enough to pass the test for significance $(0-100$ msec: $t=-1.6, P=0.111 ; 100-600$ msec: $t=-1.6, P=$ $0.122 ; n=33$ ). Dendritic reconstruction (Fig. 12C) reveals a large, somewhat elliptical, loosely branched arbor $(264 \times 137 \mu \mathrm{m})$, with sparse dendritic crossing, and eccentric cell body. No axon is evident. The image stack was not in a format suitable for Neurolucida rotation, but examination of the $\mathrm{z}$-axis image stack in ImageJ revealed a branching plane in IPL 5 .

A long-wavelength-depolarized spectrally biphasic C-type amacrine cell is illustrated in Figure 13. At 570 and $650 \mathrm{~nm}$ the stimulus causes depolarization, while at 330, 410, and 490 $\mathrm{nm}$, it causes hyperpolarization (Fig. 13A). This is red-green color opponency. Constantquanta amplitude spectra at stimulus onset (Fig. 13B) were fit (Eq. 1) to the 39-stimulus trial. Depending on the brightness of the constant irradiance modeled, peak sensitivity for excitation ranged from 573 to $583 \mathrm{~nm}$. The peak inhibition was seen between 438 and 439 $\mathrm{nm}$. Red cones depolarize this cell, and green cones hyperpolarize it ( Vr570 $\neq 0: t=4.9, P<$ $0.0001 ; \mathrm{Vg} 480 \neq 0, t=-2.8, P<0.01 ; n=39)$. Signals from blue and $\mathrm{UV}$ cones failed to be significant (Vb415 $\neq 0: t=1.9, P=0.07$; Vu362 $\neq 0: t=0.2, P=0.805, n=39$ ). The dendritic field is elongate (Fig. 13C). The long axis is $514 \mu \mathrm{m}$, the greatest dendritic extent seen in this study. The short axis is $130 \mu \mathrm{m}$. Dendritic branching is sparse, without overlap, and spine bearing. No axon is evident. The z-axis rotation (Fig. 13D) reveals a monostratified pattern in IPL $\mathrm{s} 5$, in the middle of IPL sublamina $b$.

\section{Waveforms, cone inputs, and stratification patterns}

In Table 1, the stratification patterns, dendritic field sizes, and patterns of red, green, blue, or UV cone inputs are summarized for each of five physiological groups. The study includes 36 microelectrode stains. Microelectrode encounter was most frequent among the ON-OFF and ON-OFF-Sustained groups ( $n=13$ and $n=8$, respectively). Encounter was least common 
among OFF and color-opponent types ( $n=4$ each). ON types were intermediate in occurrence $(n=7)$.

\section{Dendritic stratification patterns}

$\mathrm{ON}-\mathrm{OFF}$ and $\mathrm{ON}-\mathrm{OFF}-$ Sustained amacrine cells were similar in stratification patterns (Table 1). Of the combined 21 examples, 18 were bistratified, and only 3 were monostratified. No other physiological group included bistratified cells. Among the bistratified group, $a \& b$ bistratification $(n=14)$ was by far the most common pattern, with bistratification within sublamina a ( $\&$ a bistratification; $n=4$ ) being less common. A few in this group $(n=3)$ were monostratified, but only in sublamina a, with two in $\mathrm{s} 3$, near the $a / b$ border. Considering all ON-OFF and ON-OFF-Sustained cells, the most common sublamina a branching strata were s1 $(n=12)$ and $\mathrm{s} 2(n=7)$. In sublamina $b, \mathrm{~s} 4(n=11)$ was the most common branching stratum, with s5 arborizations seen only 3 times, and with s6 branching not seen. Mean percentage depth of stratification within the IPL is another index of stratification depth. For $a \& b$ bistratified ON-OFF cells, this was $17 \% \pm 7 \%$ for the sublamina $a$ stratum and $59 \% \pm 8 \%$ for the sublamina $b$ stratum (means $\pm \mathrm{SD}, n=14$ ). For the $a \&$ a bistratified group the mean depths were $9 \% \pm 6 \%$ and $34 \% \pm 5 \%$, respectively ( $n=$ $4)$, and for the a monostratified group, the branching depth was $32 \% \pm 6 \%(n=3)$. Mean dendritic field area for the $a \& b$ bistratified pattern was $0.041 \pm 0.039 \mathrm{~mm}^{2}(n=14$, mean \pm $\mathrm{SD})$, for the $a \&$ a bistratified pattern, $0.025 \pm 0.018 \mathrm{~mm}^{2}(n=4)$, and for the $a$ monostratified pattern, $0.013 \pm 0.003 \mathrm{~mm}^{2}(n=3)$. Table 1 provides the range of maximal dendritic widths seen in these same ON-OFF and ON-OFF-Sustained branching types.

$\mathrm{ON}$-sustained amacrine responses were characterized by a transient initial excitation at stimulus onset followed by sustained excitation during the light stimulus. No excitation transient at stimulus offset was observed in these cells. This response pattern occurred only in monostratified cells (Table 1) with processes in the mid-IPL, at the separation between $a$ and $b$ sublaminae: six of seven were monostratified in s4 (sublamina $b$ ), and the remaining one was monostratified in $\mathrm{s} 3$ (sublamina $a$ ). The fractional IPL depth was $56 \% \pm 6 \%$ (mean $\pm \mathrm{SD}, n=7$ ). The mean dendritic area in this group was $0.032 \pm 0.023 \mathrm{~mm}^{2}$ (mean $\pm \mathrm{SD}, n=$ $7)$.

The basic OFF physiology in zebrafish amacrine cells consisted of hyperpolarization at stimulus onset and depolarization at stimulus offset, a temporally two-component pattern. The sustained response during the stimulus was either continued hyperpolarization, or a depolarizing, membrane repolarization. This later case produces a three-component temporal pattern. OFF cells were all monostratified in sublamina $a$, with three occupying s1, and one arborizing in s2. The mean branching depth was $13 \% \pm 5 \%$ of IPL depth (mean $\pm \mathrm{SD}, n=$ 4). The mean dendritic area in this group was $0.034 \pm 0.020 \mathrm{~mm}^{2}$ (mean $\pm \mathrm{SD}, n=4$ ).

C-type amacrines were spectrally multiphasic sustained-response types depolarized by some wavelengths and hyperpolarized by others. Offset responses were always repolarizations in this group (no ON-OFF types). All C-types were monostratified in sublamina $b$, with three branching in $\mathrm{s} 5$ and one ramifying in $\mathrm{s} 4$. This group arborized closer to the ganglion cell bodies than any other amacrine cell group in the microelectrode dataset, with a mean IPL branching depth of $71 \% \pm 8 \%$ (mean $\pm \mathrm{SD}, n=4$ ), although 3 of the $14 a \& b$ bistratified 
ON-OFF cells also had branching planes at this depth. The mean dendritic area was 0.036 $\pm 0.018 \mathrm{~mm}^{2}(n=4$, mean $\pm \mathrm{SD})$.

\section{Spectral signals}

The signal analysis provided by Equation 1 gives parameter values for the saturated cone voltages (Vr570, Vg480, Vb415, and Vu362) impinging on amacrine cells from red, green, blue, and UV cones, respectively. For a particular cell, if any of these maximal voltage values were found to be significantly different from 0 , a $D$ or $H$ (depolarizing or hyperpolarizing) was scored for that cone input in that cell (Table 1).

In the ON-OFF and ON-OFF-Sustained groups, cumulatively, 20 of the 21 cells scored positive for significant red cone (Ar570) depolarizing input into both onset and offset transients. For the one remaining case, the trial was incomplete. Lesser signals from other cones were sometimes seen. In four cases, depolarizing onset input was also found for green or blue cones. In another four cases, a hyperpolarizing influence was fit for UV cones. About the same admixture of other cone signals was found in the offset transients (four cells with green cone depolarization, two cells with blue cone depolarization). Typically, however, $\mathrm{ON}-\mathrm{OFF}$ and ON-OFF-Sustained amacrine cells were entirely red cone driven, in all of the three stratification variants, and in both response components. The median spectral peak of the onset transient was $565 \mathrm{~nm}$, and that of the offset transient was $567 \mathrm{~nm}$ (Table 1).

ON-sustained amacrine responses, with transient depolarization at light onset and a sustained depolarization during the light stimulus, revealed a median peakresponse wavelength of $500 \mathrm{~nm}(n=7)$, suggesting a cone input pattern different from the ON-OFF grouping. The cone component analysis (Table 1) shows that all seven of these cells received significant depolarization from red cones; however, five of them were also significantly depolarized by green cones. The 500-nm median spectral peak $(n=7)$ is intermediate between the absorbance maxima for red (Ar570) and green (Ag480) pigments, suggesting mixed synergistic red and green cone depolarization, with the green cone signal being large enough to shift the spectral maxima close to the green cone absorbance peak. Blue and UV cone signals did not contribute significant inputs to the ON-sustained group.

For OFF amacrine cells, the onset and offset spectra were often different. The median wavelength peak for the onset response was $532 \mathrm{~nm}$ (Table 1). Cone component analysis (Eq. 1) indicated that all four cells in this group were hyperpolarized at ON by red cone signals, and in addition, three of them were also hyperpolarized at $\mathrm{ON}$ by green or blue cone signals. The shifting of the median spectral peak to a position intermediate between red (Ar570) and green (Ag480) absorbance peaks indicates that a combination of green and red cone signals is common within the onset hyperpolarization. The median wavelength peak for the offset spectra was $565 \mathrm{~nm}$. The depolarizing offset transient of OFF amacrine cells was in all cases dominated by red cone signals (Ar570).

Color-opponent amacrine cells always recognize more than one cone signal (Table 1), and at least one cone signal must be depolarizing and another hyperpolarizing. The spectra are multiphasic. In the four coloropponent recordings of the current study, depolarizing and hyperpolarizing signals from all cone types were seen, with the exception of UV-cone 
hyperpolarization. The depolarizing and hyperpolarizing signals in C-type cells tend to be sustained during stimulation, but with transients at wavelengths between depolarizing and hyperpolarizing spectral regions.

Red-green biphasic responses $(n=2)$, were depolarized by red cones and hyperpolarized by green cones, in one case in combination with blue cones. UV-cone signals were avoided (Table 1). The median spectral peak for depolarization was $573 \mathrm{~nm}$, and the median peak for hyperpolarization was $451 \mathrm{~nm}$. Unlike synergistic depolarizing inputs, where spectral peaks lie intermediate between the two cone absorbance peaks, opposing inputs drive the red peak to longer wavelengths than the Ar570 peak, and to shorter wavelengths than the Ag480 peak. The blue-yellow biphasic amacrine response was hyperpolarized by red and green cones and depolarized by blue and UV cones. In this case, the 507-nm hyperpolarizing spectral peak lies between the red (Ar570) and green (Ag480) absorbance peaks. Similarly, the depolarizing spectral peak at $378 \mathrm{~nm}$ is intermediate between the blue (Ab415) and UV (Au362) absorbance peaks (Table 1).

The UV-blue-green triphasic cell (Table 1) is depolarized by both UV and green cones, and hyperpolarized by blue cones. Considering the RMS signal in the first $300 \mathrm{msec}$ of the response (Table 1), there are depolarizing peaks at $359 \mathrm{~nm}$ and at $484 \mathrm{~nm}$. An inhibitory trough occurs at $412 \mathrm{~nm}$. As shown above, spectral shape depends on the response time segment measured.

\section{DISCUSSION}

\section{Stratification and physiology}

The propositions that zebrafish amacrine types process cone signals indiscriminately, and that their physiologies are not associated with patterns of dendritic arborization, are both rejected in this study. We group zebrafish amacrine cells into five physiological types, based on response waveforms for spectral stimuli. Table 1 and the circuitry diagram of Figure 14 summarize the dendritic stratification patterns and cone signals found in each physiological group. There are five monostratified (s1-s5) and two bistratified ( $a \& a, a \& b$ ) branching patterns for amacrine cells in this study, a total of seven. For ON-OFF and ON-OFFSustained physiologies, one cell was monostratified in s2 and two in s3. Four were $a \& a$ bistratified, and 14 were $a \& b$ bistratified. The hypothesis that the combined ON-OFF groups are uniformly distributed, three for each pattern, among the seven branching patterns observed for all amacrines can be rejected $\left(\chi^{2}=51.33, P<0.0001, \mathrm{df}=6\right)$. Similarly, the hypothesis that $\mathrm{ON}$ physiologies (one branching in $\mathrm{s} 3$, and six in $\mathrm{s} 4$ ) are evenly distributed, one apiece among the seven branching morphologies, can be rejected $\left(\chi^{2}=30.00, P<\right.$ $0.0001, \mathrm{df}=6$ ), as can the random branching of OFF physiologies (three branching in s1, and one in s2) and C-type physiologies (three branching in s5, and one in s4). For these latter groups, $P<0.05\left(\chi^{2}=13.51, \mathrm{df}=6\right)$. The distribution of cone signals among amacrine physiologies is far from uniform. There are two transient depolarizations for ON-OFF cells, either one of which might be driven by various combinations of signals from the four cone types. In fact, 20 of 20 onset transients, and 20 of 20 offset transients contain red cone signals. We count 15 potential depolarizing cone-signal combinations that might drive these transients (the 16th lacks signals). Red cone depolarizations occur in eight of these (53\%). 
The likelihood of a chance association between all 40 of the onset and offset transients and red cone signals is small $\left(0.53^{40}, P<0.0001\right)$. Similar to ON-OFF cells, OFF physiologies include red cone signals in four of four onset hyperpolarizing, and four of four offset depolarizing components, an unlikely chance association $\left(0.53^{8}, P<0.01\right)$. The singlecomponent $\mathrm{ON}$ physiologies included a red cone signal in all seven examples $\left(0.53^{7}, P<\right.$ 0.05); interestingly, they also exclude both blue and UV signals. We count that 7 of the 15 signal combinations (47\%) lack a cone type. The probability that two would be lacking from the seven $\mathrm{ON}$ cells is low $\left(0.47^{14}, P<0.0001\right)$. The $\mathrm{C}$-type physiology uniquely requires at least one depolarizing and one hyperpolarizing signal among combinations of four cone signals. Considering three possibilities for each of four cone signals (depolarizing, hyperpolarizing, or absent), there are 80 responsive combinations. We count 30 nonopponent and 50 opponent among them. That two high-significance $(\gg 3) \mathrm{UV}$ depolarizations occurred in the four-cell C-type group, but only one occurred in the 32-cell group including all other types suggests association between UV signals and the C-type group $\left(\chi^{2}=9.37, P<0.005, \mathrm{df}=1\right)$. Considering the rejection of the null hypotheses, we propose a functional organization for zebrafish IPL.

Amacrine cells with ON type physiology, most of them combining red and green cone signals in a sustained waveform, stratify in a single mid-IPL plane, usually sublamina $b, \mathrm{~s} 4$ (Fig. 14B, ON), but sometimes sublamina a, s3 (Fig. 14A, ON). Amacrine cells with transient depolarization at stimulus onset and offset (Fig. 14C-E, ON-OFF), irrespective of sustained components, were bistratified in sublaminas $a$ and $b$, bistratified within sublamina $a$, or monostratified in sublamina $a$. The ON-OFF physiologies were driven by signals from red cones, both at onset and offset. OFF-type amacrine cells were hyperpolarized at stimulus onset and depolarized at stimulus offset. A later depolarizing wave during stimulation occurred in some of these cells. As a group, these types received mixed signals from both red and from blue or green cones at onset, but only red cone signals at offset. The two OFF branching patterns, in s1 and s2 of sublamina a, are illustrated in Figure 14F,G.

In calcium imaging studies (Rosa et al., 2016), zebrafish amacrine cells, labeled with the transgenes ptf1a:-gal4:UAS:SyGCaMP3, all expressed calcium reporter (Jusuf and Harris, 2009). The IPL positions of those amacrine dendritic pixels showing calcium activation by 590-nm light stimuli were plotted against IPL depth for ON, OFF, and ON-OFF patterns. While pixels corresponding to all three physiologies could be found in any IPL stratum, ON activation was reported to peak in $\mathrm{s} 4$ and s5, OFF activation in s1, with a lesser peak in s4, and $\mathrm{ON}-\mathrm{OFF}$ activation, in s2 and s5. The distribution of activated pixels in these 10-dayold larvae is compatible with the physiologies associated with dendritic branching planes seen in adult ON, OFF, and ON-OFF amacrine cells studied by microelectrodes (Fig. 14).

C-type amacrine cells are a spectrally heterogeneous group but have in common multiphasic spectra. In this group, different wavelengths evoke different waveforms, characterized by depolarization for some wavelengths and hyperpolarization for others. These types have in common a monostratified dendritic arbor in $\mathrm{s} 4$ or $\mathrm{s} 5$ of sublamina $b$ (Fig. 14H). C-type arbors lay the farthest from the amacrine cell border of the IPL of any physiological group in this dataset. 


\section{Microelectrode classifications versus anatomical classifications}

Amacrine-cell dendritic branching in zebrafish has been studied by morphological methods, including gene-gun sprays with Dil pellets (Connaughton et al., 2004; Connaughton and Hsieh, 2008), antibodies (Connaughton et al., 1999; Yazulla and Studholme, 2001; Arenzana et al., 2005; Yeo et al., 2009; Jang et al., 2011), and selective transgene insertions (Godinho et al., 2005; Jusuf and Harris, 2009; Nelson et al., 2009; Lewis et al., 2015). While all these methods suggest functional stratification bands for amacrine dendrites within the IPL, each comes with both known and unknown selection biases. In the present microelectrode study, no displaced types were encountered, whereas anti-parvalbumin and anti- $\gamma$-aminobutyric acid (GABA) immunoreactivity label displaced amacrines in adult zebrafish (Connaughton et al., 1999; Yeo et al., 2009), as does the ptfla reporter, an interneuron marker in larvae (Jusuf and Harris, 2009). On the other hand, the ptfla reporter line labels few bistratified amacrine cells ( 2 of 175), whereas microelectrodes encounter many (21 of 36). Interestingly, s1/s5 bistratified amacrine cells are immunoreactive for tyrosine hydroxylase (Jang et al., 2011), raising the likely possibility that some zebrafish dopaminergic amacrine cells are ON-OFF types. In an adult study that involved the spraying of Dil pellets on retinal slices (Connaughton et al., 2004), monostratified amacrine types were found occupying practically every stratum in the IPL. A single bistratified type (s1/s5) was illustrated. In a later study (Connaughton and Hsieh, 2008), OFF branching, ON branching, and $a \& b$ bistratified types were found in about equal numbers. In that study, similar to the present data, some bistratified amacrine cells were found within the confines of sublamina a. However, in slice staining, dendritic field extents were about half that reported in the current dataset. While the live slice preparation (Connaughton, 2003) provides good access for the gene-gun pellets to label amacrine cells, and an excellent view of stratification, amacrine fields are elliptical, and the long axis need not orient in the direction of slice, leading to underestimates of dendritic extent.

Connaughton et al. (2004) noted four monostratified types branching in s1, s2, s4, and s5. The s1 and s2 types were termed "OFF" types in accordance with the sublamina a arborization. This anatomical inference is verified in four of five microelectrode stains, the exception being a lone $\mathrm{ON}-\mathrm{OFF}$ cell monostratified in s2. The s4 and $\mathrm{s} 5$ types were previously termed "ON" types (Connaughton et al., 2004). These types also appear in the present microelectrode stains. Connaughton et al. (2004) even point out the spines on the s5 type, which can also be seen in the microelectrode stains of Figures 11-13. While the s4 monostratified type is indeed ON type in physiology, the s5 monostratified type is likely to be "C-type" based on the present microelectrode recordings. The $a \& b$ bistratified s1/s5 "ON type" (Connaughton et al., 2004) is surely an ON-OFF cell. The Dil technique also labeled diffuse types (Connaughton et al., 2004), not encountered by the microelectrode in the current dataset.

In the present recordings, neither dendritic area nor the lateral microstructure of dendritic branching seemed significantly related to either the waveform of the light response, or the cone signal composition of the response. Only dendritic stratification patterns correlated with physiology. This leads to only a small inventory of structural properties that could be predicted from physiology. In the roach, like the zebrafish a cyprinid, considering these 
other anatomical features, 43 amacrine geometries were identified (Wagner and Wagner, 1988). These other features of lateral extent and branching structure might relate to functional properties that we did not examine.

Mammalian amacrine architecture differs from that of the zebrafish. While in this study half the amacrine types encountered by the microelectrode were bistratified types, in rabbit retina, only $1 \%$ of amacrine cells were the corresponding "planar bistratified" type (MacNeil et al., 1999). In a comprehensive study of Golgistained retinal neurons in cat, no "planar bistratified" morphologies were encountered among the 22 amacrine types described (Kolb et al., 1981). In the cat, one rarely encountered, physiologically ON-OFF amacrine cell has been reported, the s2 monostratified A19 type (Freed et al., 1996). It seems possible that the ON-OFF motion-sensing pathway represented by widefield bistratified amacrine cells of fish (Kaneko, 1973) and reptiles (Schwartz, 1973) is largely absent in mammals.

\section{Signal sources in a physiologically laminated IPL}

The restricted stratification planes of zebrafish amacrine-cell dendrites make them reporters of function within strata, both as signal contributors and as signal recipients. Amacrine cells provide GABAergic or glycinergic inhibition of other amacrine, bipolar, and ganglion cells (Pang et al., 2002; Rosa et al., 2016). ON-OFF and ON-OFF-Sustained amacrine cells would likely provide transient inhibitory signals from red cones to IPL strata 1-5. For ONOFF cells restricted in branching to sublamina a, both the ON and the OFF transients must arise from synapses within sublamina a. SyGCaMP2 calcium imaging of zebrafish bipolar terminals reveals five to six physiological types defined by ON or OFF pattern, frequency response, and the presence or absence of spiking (Baden et al., 2011; Dreosti et al., 2011; Rosa et al., 2016). As measured by calcium activation, both ON and OFF bipolar physiologies are reported throughout sublamina $a$, with peaks for OFF type 3 (highfrequency) boutons in s1, and ON contrast-suppressed boutons in s3 (Rosa et al., 2016). An individual patch recording of an ON bipolar cell with a terminal in s1 was reported in goldfish (Baden et al., 2011). We speculate that these sublamina a ON and OFF bipolar terminals innervate sublamina $a \mathrm{ON}-\mathrm{OFF}$ amacrine types. The s1 OFF terminals (Rosa et al., 2016) are also well positioned to innervate the s1 or s2 stratified OFF amacrines.

Traditionally, ON-OFF $a / b$ bistratified amacrine cells are thought to receive OFF signals from sublamina $a$, and ON signals from sublamina $b$. Indeed, a prominent band of ON contrast-activated bipolar boutons was reported in s5 (Rosa et al., 2016). This might supply the ON signals for $a / b$ bistratified amacrine cells (Fig. 14D); however, the availability of ON signals in more distal strata makes this conclusion uncertain. A further uncertainty is the source of signals for the s4-stratifying ON-type amacrines with sustained responses. This stratum appears to contain few glutamatergic bipolar terminals (Connaughton et al., 2004), and is almost devoid of light-sensitive calcium activation, but does lie between strata s3 and s5, which show peaks in ON-type bipolar-terminal distribution (Rosa et al., 2016).

The zebrafish IPL differs from the standard mammalian model, in which only OFF bipolar cells make synapses in sublamina $a$, while ON bipolar cells traverse this layer with axons of passage to synapse in sublamina $b$ (Nelson et al., 1978; Kolb, 1979). Axon terminals of zebrafish bipolar cells can be either monostratified within a single sublamina, or bistratified 
in both (Connaughton et al., 2004; Wong and Dowling, 2005; Li et al., 2012). The finding of ON-OFF amacrine cells arborizing within sublamina a could be supported by ON-type bipolar cells with terminals bistratified in sublaminas $a$ and $b$, or even, perhaps, ON-type bipolar cells terminating in sublamina $a$ (Baden et al., 2011). Nonetheless, zebrafish ON and OFF amacrine types do appear to be traditionally placed within the IPL sublaminas.

Another interesting stratum is s5, the branching level for C-type amacrine cells. In mammals, this stratum contains the axon terminals of bipolar cells devoted to shortwavelength cones (Mariani, 1984; Euler et al., 1996; Haverkamp et al., 2005; Breuninger et al., 2011). In primates, short-wavelength cones are color opponent (Packer et al., 2010). Color opponency arises through horizontal cell feedback onto cones (Stell and Lightfoot, 1975), a mechanism common to vertebrates. In zebrafish, like primates, short-wavelength cones may become color opponent through feedback (Li et al., 2009; Connaughton and Nelson, 2010). Red cone signals, as represented in H1 horizontal cells, confer biphasic (red depolarizing, blue and green hyperpolarizing) physiology onto blue cones. H2 horizontal cells reflect this blue-cone biphasic pattern, and feed back onto UV cones, creating a triphasic physiology. In this model, these color-opponent short-wavelength signals from blue and UV cones feed into s5, deep in the IPL, through the axon terminals of cone bipolar cells that represent these short-wavelength cone signals, and introduce their multiphasic spectral physiology into this stratum.

Li et al. (2012) suggest that the major bipolar-cell input to $s 5$ is provided by bistratified s3/s5 (Cab) bipolar cells that contact green, blue, and UV cones. These might be the relatives of the mammalian "blue-cone" bipolar cell. Analogous to the mammalian homologue, $\mathrm{Cab}$ is the only cone bipolar type that contacts short-wavelength cones, but excludes contact with long-wavelength cones. In defense of this proposed homology, the RH2 and SWS2 (green and blue) cone/opsin types that Cab cells also contact in zebrafish do not exist in mammals. Only the SWS1 (UV) opsin is homologous (Vihtelic et al., 1999).

In giant danio, a cyprinid fish similar to zebrafish, bistratified, s3/s5 Cabs hyperpolarized on dendritic application of D-aspartate, but depolarized on dendritic application of kainate (Wong and Dowling, 2005). Cab center mechanisms were often found to be color opponent, and the receptor physiology suggested the possibility of direct generation of spectrally multiphasic responses through cone-selective expression of opposing postsynaptic glutamate mechanisms (Wong and Dowling, 2005). Postsynaptic gating of AMPA/kainate-driven cation channels by glutamate released from one cone type might be pitted against postsynaptic EAAT5 glutamate transporters, which form chloride channels on binding glutamate released from another cone type. Opposing membrane polarities would be evoked by different cones. Cab cells then may generate color opponent signals by two mechanisms: 1) color-opponent signals inherited from feedback; and 2) cone-selective postsynaptic mechanisms on bipolar cells.

The single Cab morphology can represent several different color-opponent patterns (Wong and Dowling, 2005). Similarly, the zebrafish type H3 retinal horizontal cell has three variants of color-opponent organization (Connaughton and Nelson, 2010), but only a single morphology and photoreceptor connectivity (Song et al., 2008; Li et al., 2009). In addition 
to morphological patterns of cone contact, spectral physiology appears to depend on different patterns of postsynaptic receptor expression and corresponding signal integration. In this model, different spectral types of C-type amacrine cells may need to discriminate Cab bipolar cell axon boutons with different spectral physiologies.

\section{Acknowledgments}

This research was supported by the Intramural Research Program of the National Institute of Neurological Disorders and stroke, NIH, and the Intramural Research Program of the National Institute of Alcoholism and Alcohol Abuse, NIH.

We thank D. Graham, J. Urban, and T. Ikenaga for contributions to this study.

\section{LITERATURE CITED}

Ammermüller J, Muller JF, Kolb H. The organization of the turtle inner retina. II. Analysis of colorcoded and directionally selective cells. J Comp Neurol. 1995; 358:35-62. [PubMed: 7560276]

Arenzana FJ, Clemente D, Sánchez-González R, Porteros Á, Aijón J, Arévalo R. Development of the cholinergic system in the brain and retina of the zebrafish. Brain Res Bull. 2005; 66:421-425. [PubMed: 16144624]

Avdesh A, Martin-Iverson MT, Mondal A, Chen M, Askraba S, Morgan N, Lardelli M, Groth DM, Verdile G, Martins RN. Evaluation of color preference in zebrafish for learning and memory. $\mathbf{J}$ Alzheim Dis. 2012; 28:459-469.

Baden T, Esposti F, Nikolaev A, Lagnado L. Spikes in retinal bipolar cells phase-lock to visual stimuli with millisecond precision. Curr Biol. 2011; 2:1859-1869.

Barlow HB, Levick WR. The mechanism of directionally selective units in rabbit's retina. J Physiol. 1965; 178:477-504. [PubMed: 5827909]

Breuninger T, Puller C, Haverkamp S, Euler T. Chromatic bipolar cell pathways in the mouse retina. J Neurosci. 2011; 31:6504-6517. [PubMed: 21525291]

Briggman KL, Helmstaedter M, Denk W. Wiring specificity in the direction-selectivity circuit of the retina. Nature. 2011; 471:183-188. [PubMed: 21390125]

Burkhardt DA. Zebrafish inner retina: local signals for spatial position, luminance, and color contrast. Vis Neurosci. 2012; 29:229-236. [PubMed: 22877609]

Chen S, Li W. A color-coding amacrine cell may provide a blue-off signal in a mammalian retina. Nat Neurosci. 2012; 15:954-956. [PubMed: 22634731]

Chinen A, Hamaoka T, Yamada Y, Kawamura S. Gene duplication and spectral diversification of cone visual pigments of zebrafish. Genetics. 2003; 163:663-675. [PubMed: 12618404]

Colwill RM, Raymond MP, Ferreira L, Escudero H. Visual discrimination learning in zebrafish (Danio rerio). Behav Proc. 2005; 70:19-31.

Connaughton VP. Zebrafish retinal slice preparation. Methods Cell Sci. 2003; 25:49-58. [PubMed: 14739587]

Connaughton VP, Hsieh J. Morphological classification of amacrine cells in the zebrafish retina. Invest Ophthalmol Vis Sci. 2008; 49:5905.

Connaughton VP, Nelson R. Spectral responses in zebrafish horizontal cells include a tetraphasic response and a novel UV-dominated triphasic response. J Neurophysiol. 2010; 104:2407-2422. [PubMed: 20610786]

Connaughton VP, Behar TN, Liu WL, Massey SC. Immunocytochemical localization of excitatory and inhibitory neurotransmitters in the zebrafish retina. Vis Neurosci. 1999; 16:483-490. [PubMed: 10349969]

Connaughton VP, Graham D, Nelson R. Identification and morphological classification of horizontal, bipolar, and amacrine cells within the zebrafish retina. Journal of Comparative Neurology. 2004; 477:371-385. [PubMed: 15329887] 
Dowling JE, Werblin FS. Organization of retina of the mudpuppy, Necturus maculosus. I. Synaptic structure. J Neurophysiol. 1969; 32:315-338. [PubMed: 5787842]

Dreosti E, Esposti F, Baden T, Lagnado L. In vivo evidence that retinal bipolar cells generate spikes modulated by light. Nat Neurosci. 2011; 14:951-952. [PubMed: 21706020]

Endeman D, Klaassen LJ, Kamermans M. Action spectra of zebrafish cone photoreceptors. PloS One. 2013; 8:e68540. [PubMed: 23861916]

Engstrom K. Cone types and cone arrangement in the retina of some cyprinids. Acta Zool. 1960; 41:277-295.

Euler T, Schneider H, Wässle H. Glutamate responses of bipolar cells in a slice preparation of the rat retina. J Neurosci. 1996; 16:2934-2944. [PubMed: 8622124]

Euler T, Detwiler PB, Denk W. Directionally selective calcium signals in dendrites of starburst amacrine cells. Nature. 2002; 418:845-852. [PubMed: 12192402]

Freed MA, Pflug R, Kolb H, Nelson R. ON-OFF amacrine cells in cat retina. J Comp Neurol. 1996; 364:556-566. [PubMed: 8820883]

Godinho L, Mumm JS, Williams PR, Schroeter EH, Koerber A, Park SW, Leach SD, Wong RO. Targeting of amacrine cell neurites to appropriate synaptic laminae in the developing zebrafish retina. Development. 2005; 132:5069-5079. [PubMed: 16258076]

Gouras P. Identification of cone mechanisms in monkey ganglion cells. J Physiol. 1968; 199:533-547. [PubMed: 4974745]

Halloran MC, Sato-Maeda M, Warren JT, Su F, Lele Z, Krone PH, Kuwada JY, Shoji W. Laser-induced gene expression in specific cells of transgenic zebrafish. Development. 2000; 127:1953-1960. [PubMed: 10751183]

Haverkamp S, Wässle H, Duebel J, Kuner T, Augustine GJ, Feng G, Euler T. The primordial, bluecone color system of the mouse retina. J Neurosci. 2005; 25:5438-5445. [PubMed: 15930394]

Hughes A, Saszik S, Bilotta J, Demarco PJ Jr, Patterson WF 2nd. Cone contributions to the photopic spectral sensitivity of the zebrafish ERG. Vis Neurosci. 1998; 15:1029-1037. [PubMed: 9839967]

Ikenaga T, Urban JM, Gebhart N, Hatta K, Kawakami K, Ono F. Formation of the spinal network in zebrafish determined by domain-specific pax genes. J Comp Neurol. 2011; 519:1562-1579. [PubMed: 21452218]

Jang YJ, Yu SH, Lee ES, Jeon CJ. Two types of tyrosine hydroxylase-immunoreactive neurons in the zebrafish retina. Neurosci Res. 2011; 71:124-133. [PubMed: 21784111]

Jusuf PR, Harris WA. Ptf1a is expressed transiently in all types of amacrine cells in the embryonic zebrafish retina. Neural Dev. 2009; 4:8104-8104.

Kaneko A. Receptive field organization of bipolar and amacrine cells in the goldfish retina. J Physiol. 1973; 235:133-153. [PubMed: 4778132]

Kawakami K, Takeda H, Kawakami N, Kobayashi M, Matsuda N, Mishina M. A transposon-mediated gene trap approach identifies developmentally regulated genes in zebrafish. Dev Cell. 2004; 7:133-144. [PubMed: 15239961]

Kolb H. The inner plexiform layer in the retina of the cat: electron microscopic observations. J Neurocytol. 1979; 8:295-329. [PubMed: 490185]

Kolb H, Famigilietti E. Rod and cone pathways in the inner plexiform layer of cat retina. Science. 1974; 186:47-49. [PubMed: 4417736]

Kolb H, Nelson R, Mariani A. Amacrine cells, bipolar cells and ganglion cells of the cat retina: a Golgi study. Vision Res. 1981; 21:1081-1114. [PubMed: 7314489]

Krauss A, Neumeyer C. Wavelength dependence of the optomotor response in zebrafish (Danio rerio). Vision Res. 2003; 43:1275-1284.

Lewis A, Wilson N, Stearns G, Johnson N, Nelson R, Brockerhoff SE. Celsr3 is required for normal development of GABA circuits in the inner retina. PLoS Genet. 2011; 7:e1002239. [PubMed: 21852962]

Lewis AA, Mahoney JT, Wilson N, Brockerhoff SE. Identification of amacrine subtypes that express the atypical cadherin celsr3. Exp Eye Res. 2015; 130:51-57. [PubMed: 25479044]

Li YN, Matsui JI, Dowling JE. Specificity of the horizontal cell-photoreceptor connections in the zebrafish (Danio rerio) retina. J Comp Neurol. 2009; 516:442-453. [PubMed: 19655401] 
Li YN, Tsujimura T, Kawamura S, Dowling JE. Bipolar cell-photoreceptor connectivity in the zebrafish (Danio rerio) retina. J Comp Neurol. 2012; 520:3786-3802. [PubMed: 22907678]

MacNeil MA, Heussy JK, Dacheux RF, Raviola E, Masland RH. The shapes and numbers of amacrine cells: matching of photofilled with Golgi-stained cells in the rabbit retina and comparison with other mammalian species. J Comp Neurol. 1999; 413:305-326. [PubMed: 10524341]

Mariani AP. Bipolar cells in monkey retina selective for the cones likely to be blue-sensitive. Nature. 1984; 308:184-186. [PubMed: 6199677]

Mills SL, Tian L-M, Hoshi H, Whitaker CM, Massey SC. Three distinct blue-green color pathways in a mammalian retina. J Neurosci. 2014; 34:1760-1768. [PubMed: 24478358]

Mitarai G, Goto T, Takagi S. Receptive field arrangement of color-opponent bipolar and amacrine cells in the carp retina. Sens Proc. 1978; 2:375-382.

Müller F, Wässle H, Voigt T. Pharmacological modulation of the rod pathway in the cat retina. J Neurophysiol. 1988; 59:1657-1672. [PubMed: 3404200]

Nelson R. AII amacrine cells quicken time course of rod signals in the cat retina. J Neurophysiol. 1982; 47:928-947. [PubMed: 6177841]

Nelson R, Kolb H. A17: a broad-field amacrine cell in the rod system of the cat retina. J Neurophysiol. 1985; 54:592-614. [PubMed: 4045539]

Nelson RF, Singla N. A spectral model for signal elements isolated from zebrafish photopic electroretinogram. Vis Neurosci. 2009; 26:349-363. [PubMed: 19723365]

Nelson R, Famiglietti EV Jr, Kolb H. Intracellular staining reveals different levels of stratification for on- and off-center ganglion cells in the cat retina. J Neurophysiol. 1978; 41:472-483. [PubMed: 650277]

Nelson RF, Ma TS, Ikenaga T, Connaughton VP, Ono F. Forward transgenic selectively Labels amacrine, ganglion, and horizontal cell types in zebrafish retina. Invest Ophthalmol Vis Sci. 2009; 50:1301.

Ono F, Higashijima S-i, Shcherbatko A, Fetcho JR, Brehm P. Paralytic zebrafish lacking acetylcholine receptors fail to localize rapsyn clusters to the synapse. J Neurosci. 2001; 21:5439-5448. [PubMed: 11466415]

Orger MB, Baier H. Channeling of red and green cone inputs to the zebrafish optomotor response. Vis Neurosci. 2005; 22:275-281. [PubMed: 16079003]

Packer OS, Verweij J, Li PH, Schnapf JL, Dacey DM. Blue-yellow opponency in primate S cone photoreceptors. J Neurosci. 2010; 30:568-572. [PubMed: 20071519]

Palacios AG, Goldsmith TH, Bernard GD. Sensitivity of cones from a cyprinid fish (Danio aequipinnatus) to ultraviolet and visible light. Vis Neurosci. 1996; 13:411-421. [PubMed: 8782369]

Pang J-J, Gao F, Wu SM. Relative contributions of bipolar cell and amacrine cell inputs to light responses of ON, OFF and ON-OFF retinal ganglion cells. Vision Res. 2002; 42:19-27. [PubMed: 11804628]

Raymond PA, Barthel LK, Curran GA. Developmental patterning of rod and cone photoreceptors in embryonic zebrafish. J Comp Neurol. 1995; 359:537-550. [PubMed: 7499546]

Robinson J, Schmitt EA, Harosi FI, Reece RJ, Dowling JE. Zebrafish ultraviolet visual pigment: absorption spectrum, sequence, and localization. Proc Natl Acad Sci U S A. 1993; 90:6009-6012. [PubMed: 8327475]

Rosa JM, Ruehle S, Ding H, Lagnado L. Crossover inhibition generates sustained visual responses in the inner retina. Neuron. 2016; 90:308-319. [PubMed: 27068790]

Schwartz E. Organization of on-off cells in the retina of the turtle. J Physiol. 1973; 230:1-14. [PubMed: 4702424]

Sher A, DeVries SH. A non-canonical pathway for mammalian blue-green color vision. Nat Neurosci. 2012; 15:952-953. [PubMed: 22634728]

Song PI, Matsui JI, Dowling JE. Morphological types and connectivity of horizontal cells found in the adult zebrafish (Danio rerio) retina. J Comp Neurol. 2008; 506:328-338. [PubMed: 18022944]

Stell WK, Lightfoot DO. Color-specific interconnections of cones and horizontal cells in the retina of the goldfish. J Comp Neurol. 1975; 159:473-502. [PubMed: 1092733] 
Tauchi M, Masland R. The shape and arrangement of the cholinergic neurons in the rabbit retina. Proc R Soc Lond B Biol Sci. 1984; 223:101-119. [PubMed: 6151180]

Vihtelic TS, Doro CJ, Hyde DR. Cloning and characterization of six zebrafish photoreceptor opsin cDNAs and immunolocalization of their corresponding proteins. Vis Neurosci. 1999; 16:571-585. [PubMed: 10349976]

Wagner HG, Macnichol EF, Wolbarsht ML. The response properties of single ganglion cells in the goldfish retina. J Gen Physiol. 1960; 43:45-62. [PubMed: 19873528]

Wagner HJ, Wagner E. Amacrine cells in the retina of a teleost fish, the roach (Rutilus rutilus): a Golgi study on differentiation and layering. Philos Trans R Soc Lond B Biol Sci. 1988; 321:263-324. [PubMed: 2906747]

Werblin FS, Dowling JE. Organization of the retina of the mudpuppy, Necturus maculosus. II. Intracellular recording. J Neurophysiol. 1969; 32:339-355. [PubMed: 4306897]

Wong KY, Dowling JE. Retinal bipolar cell input mechanisms in giant Danio: III. ON-OFF bipolar cells and their color-opponent mechanisms. J Neurophysiol. 2005; 94:265-272. [PubMed: 15758056]

Yazulla S, Studholme KM. Neurochemical anatomy of the zebrafish retina as determined by immunocytochemistry. J Neurocytol. 2001; 30:551-592. [PubMed: 12118162]

Yeo JY, Lee ES, Jeon CJ. Parvalbumin-immunoreactive neurons in the inner nuclear layer of zebrafish retina. Exp Eye Res. 2009; 88:553-560. [PubMed: 19084520] 

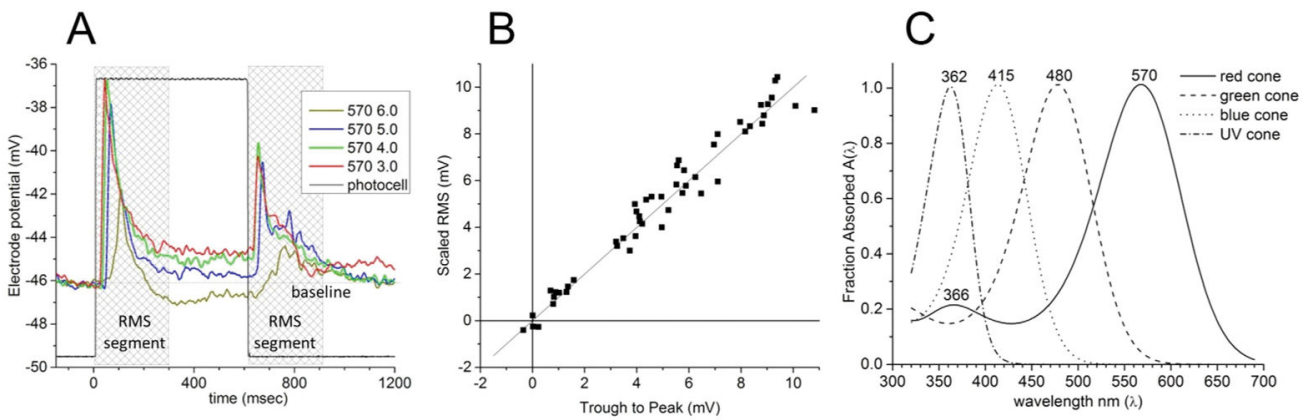

Figure 1.

Response amplitude and cone signal measurement. A: Intensity series for ON-OFF amacrine. The root-mean-square (RMS) segments (hatched) show the intervals over which the scaled RMS amplitudes are calculated. The wavelength is $570 \mathrm{~nm}$, the light step is 600 msec, and the stimulus irradiance varies from 2.0 to $5.0 \log \left(h v \cdot \mu \mathrm{m}^{-2} \cdot \mathrm{s}^{-1}\right), 6.0$ to $3.0 \log$ units of neutral density. 22Oct2007 Cell \#1 WT. B: The scaled RMS amplitude, calculated in the 300-msec segment after stimulus onset, is plotted against the trough-to-peak amplitude in the same interval for 51 stimuli applied to the cell in A. The comparison line is slope 1. To calculate RMS amplitude, the potential at each time point in the RMS interval is subtracted from baseline, squared, summed, divided by the number of points, and the square root taken. A single scale factor is applied to the raw RMS amplitude to best approximate trough-topeak amplitude. The response sign is taken from the mean in the interval with respect to baseline. C: Opsin absorbances for red, green, blue, and UV cones. These are the A( $\lambda)$ functions (Ar570, Ag480, Ab415, Au362) used in Equation 1 to determine the spectral component signals contributing to amacrine responses. The curves are derived from the nomogram of Hughes et al. (1998) for red, green, and blue opsins, and from Palacios et al. (1996) for the UV opsin. [Color figure can be viewed at wileyonlinelibrary.com] 

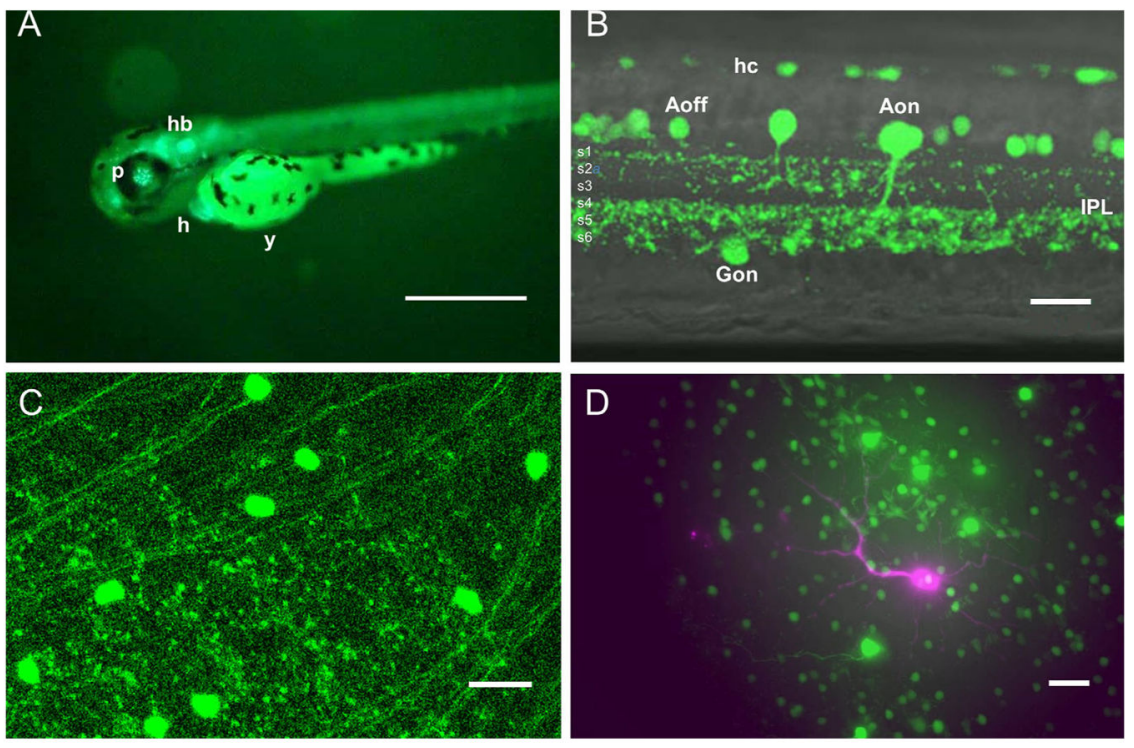

Figure 2.

GFP expression pattern in the GE4a retina. A: GE4a larva at 4 days post fertilization. GFPstained retinal neurons are seen through the pupil (p). Hindbrain structures (hb) label with GFP, as does heart (h). The yolk (y) is autofluorescent. B: Adult GE4a live retinal slice. Select populations of a horizontal cell (hc), amacrine cell (Aoff, Aon), and ganglion cell (Gon) are labeled. The IPL is subdivided into strata s1-s6 (left). The OFF layer of the IPL (sublamina $a$ ) is composed of strata s1-s3. The ON layer (sublamina $b$ ) is composed of strata s4-s6 (Connaughton et al., 2004). C: Adult GE4a live retinal eyecup, viewed en face. GFP labels a ganglion cell population, together with axons in the optic fiber layer. D:

Microelectrode-injected Alexa 594 hydrazide (magenta) stains an OFF-type amacrine cell in an adult, live, flattened GE4a eyecup. Alexa 594-stained dendrites (reconstructed in Fig. 7) course close to GFP-stained amacrine cell bodies. Image A, fluorescence binocular scope; B and D, confocal; D, widefield microscopy. Images $\mathrm{B}$ and $\mathrm{C}$ are $\mathrm{z}$-axis projections of two to four planes. Image D is a Z-axis projection of $\sim 100$ planes. Scale bar $=1 \mathrm{~mm}$ in A; 20 $\mathrm{mm}$ in B-D. [Color figure can be viewed at wileyonlinelibrary.com] 
A
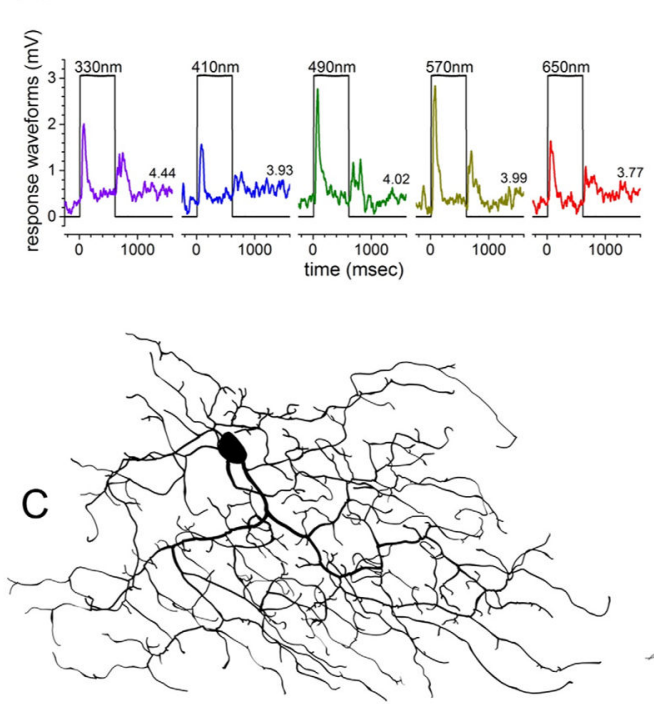

B
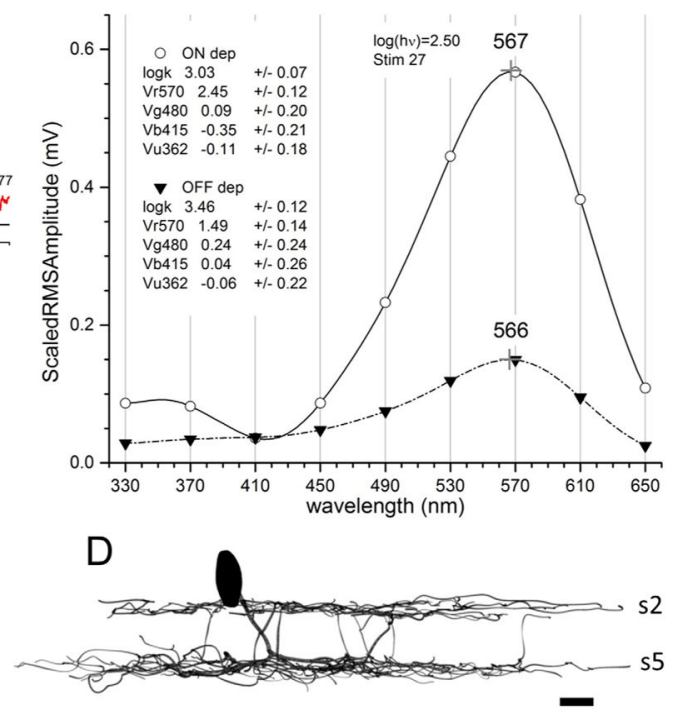

Figure 3.

ON-OFF amacrine, $a / b$ bistratified type. A: Transient depolarizations (dep) following stimulus onset and offset are evoked by wavelengths from 330 to $650 \mathrm{~nm}$. Numbers above each trace are the stimulus irradiances in $\log \left(h v \cdot \mu \mathrm{m}^{-2} \cdot \mathrm{s}^{-1}\right)$. Waveforms are filtered by a 33point (16.5-msec) running average. Penetration potential, $-33 \mathrm{mV}$. B: The spectral model (Eq. 1, 27 stimuli) is fit both at ON (ON dep, open circles) and at OFF (OFF dep, filled triangles) to the RMS amplitudes during the $300 \mathrm{msec}$ after stimulus onset, or the $300 \mathrm{msec}$ after stimulus offset. Spectral amplitudes are interpolated (Eq. 1) for constant quanta stimuli of $2.5 \log \left(h v \cdot \mu \mathrm{m}^{-2} \cdot \mathrm{s}^{-1}\right)$ at all wavelengths. Fit parameters $( \pm \mathrm{SE})$ indicate that only red cone signals are significant for $\mathrm{ON}$ and $\mathrm{OFF}$ depolarizations (Vr570 $\neq 0)$. C: Neurolucida reconstruction of the microelectrode injected cell whose responses appear in A. D: Rotation of the 3D reconstruction reveals two planes of dendritic branching in IPL s2 and s5. 16Sept2009 Cell\#1, adult GE4a. Scale bar $=10 \mu \mathrm{m}$ in C and D. [Color figure can be viewed at wileyonlinelibrary.com] 
A

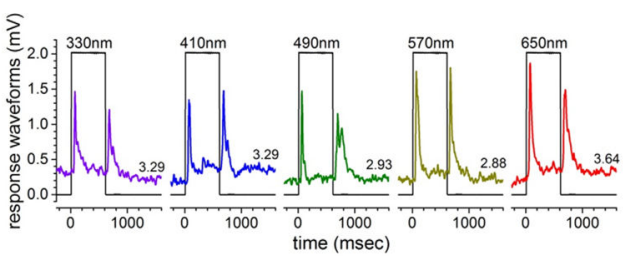

C

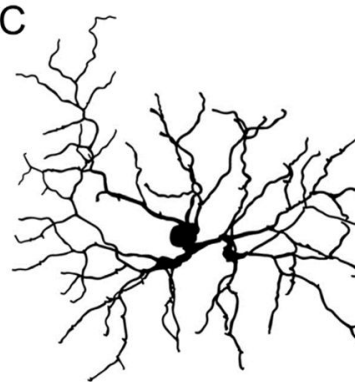

B

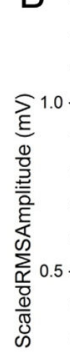

O ON dep $\begin{array}{lll}0 & \text { ON dep } \\ \text { logk } 2.36 & +/-0.06\end{array}$ $\begin{array}{lll}\mathrm{Vg} 480 & 0.10 & +-0.06 \\ +-0.11\end{array}$ $\begin{array}{lll}V \mathrm{~V} 480 & 0.10 & +/-0.11 \\ \mathrm{Vb} 415 & 0.03 & +/-0.13\end{array}$ $\begin{array}{llll}\text { Vu362 } & -0.01 & +/-0.11\end{array}$

$\checkmark$ OFF dep

$\begin{array}{ll}\text { V OFF dep } & \\ \text { logk } 2.92 & +-0.09 \\ \text { Vr570 } 1.74 & +-0.11\end{array}$

$V g 480 \quad 0.26++1-0.19$

$\begin{array}{llll} & V \\ V b 415 & 0.14 & +/-0.23\end{array}$

$\begin{array}{lll}\text { Vu362 } & 0.09 & +/-0.20\end{array}$

砶
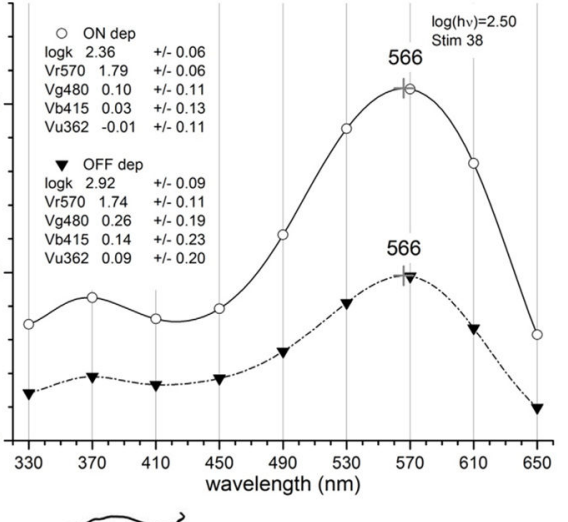

Figure 4.

Axon-bearing $\mathrm{ON}-\mathrm{OFF}$ amacrine, $a / b$ bistratified type. A: Transient depolarizing (dep) voltage changes following stimulus onset or offset are seen for all wavelengths from 330 to $650 \mathrm{~nm}$. Numbers above each trace are the stimulus irradiances in $\log \left(h \nu \cdot \mu \mathrm{m}^{-2} \cdot \mathrm{s}^{-1}\right)$. Waveforms are filtered by a 33-point $(16.5$-msec) running average. Penetration potential, $-30 \mathrm{mV}$. B: The spectral model (Eq. 1, 38 stimuli) is fit both at ON (ON dep, open circles) and at OFF (OFF dep, filled triangles) to the RMS amplitudes during the $300 \mathrm{msec}$ following onset or offset of the stimuli. The spectral curves are interpolations (Eq. 1) of response amplitudes for constant quantal stimuli at $2.5 \log \left(h v \cdot \mu \mathrm{m}^{-2} \cdot \mathrm{s}^{-1}\right)$. Fit parameters $( \pm$ $\mathrm{SE})$ indicate that only signals from red cones are significant for ON or OFF depolarizations $(\mathrm{Vr} 570 \neq 0)$. C: Neurolucida reconstruction of the cell whose responses appear in A. D: Rotation of the 3D reconstruction reveals two planes of dendritic branching in IPL s2 and s4. An axon-like process projects from the s2 arbor, and remains in s2. 13May2010 Cell\#1, adult WT. Scale bar $=10 \mu \mathrm{m}$ in $\mathrm{C}$ and D. [Color figure can be viewed at wileyonlinelibrary.com] 
A
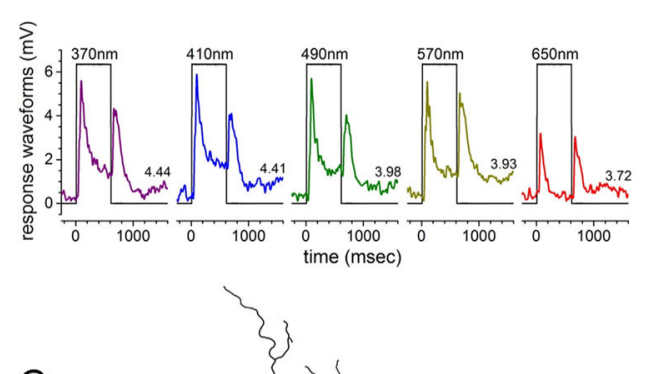

C

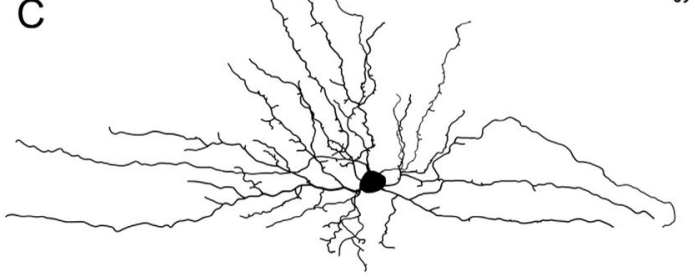

D ${ }_{s 3} 1$
B
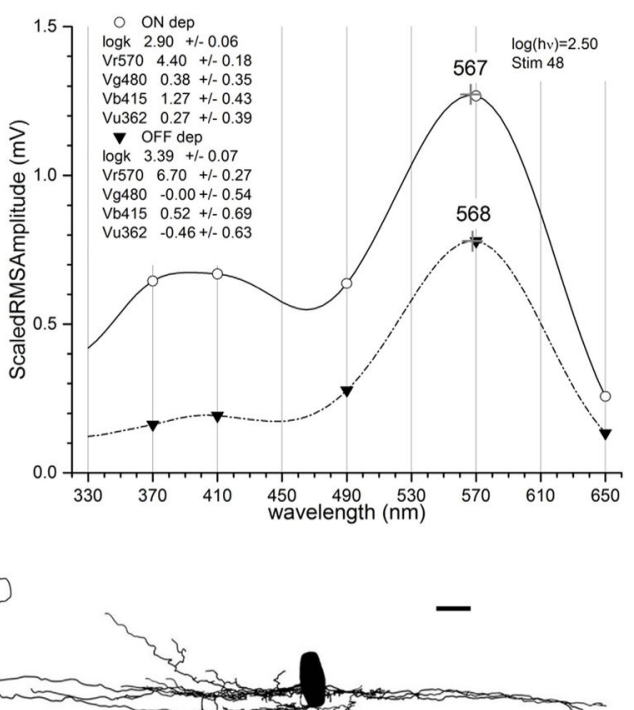

Figure 5.

ON-OFF-Sustained amacrine, bistratified in sublamina a. A. Wavelengths from 370 to 650 $\mathrm{nm}$ all evoke transient, depolarizing (dep) voltage changes following stimulus onset and offset. There is, in addition, a sustained depolarization during the stimulus. Numbers above each trace are the stimulus irradiances in $\log \left(h \nu \cdot \mu \mathrm{m}^{-2} \cdot \mathrm{s}^{-1}\right)$. Waveforms are filtered by a 33point (16.5-msec) running average. Penetration potential, $-37 \mathrm{mV}$. B: The spectral model (Eq. 1, 48 stimuli) is fit both at ON (ON dep, open circles) and at OFF (OFF dep, filled triangles) to the RMS amplitudes during the $300 \mathrm{msec}$ following onset or offset of the stimuli. The spectral curves are interpolations (Eq. 1) of response amplitudes for a constant quantal stimulus of $2.5 \log \left(h v \cdot \mu \mathrm{m}^{-2} \cdot \mathrm{s}^{-1}\right)$ at all wavelengths. Fit parameters $( \pm \mathrm{SE})$ indicate that both red and blue cones make significant contributions to the ON transient (ON dep, Vr570 $\neq 0$, Vb415 $\neq 0$ ), but that only red signals appear in the OFF transient (OFF dep, Vr570 ₹0). C: Neurolucida reconstruction of the microelectrode stained cell whose responses appear in A reveals an elongated dendritic field. D: Rotation of the 3D reconstruction reveals planes of dendritic branching in both IPL $s 1$ and s3, both within sublamina a (a \& a bistratification). 4March2010 Cell \#4, adult WT. Scale bar $=20 \mu \mathrm{m}$ in C and D. [Color figure can be viewed at wileyonlinelibrary.com] 

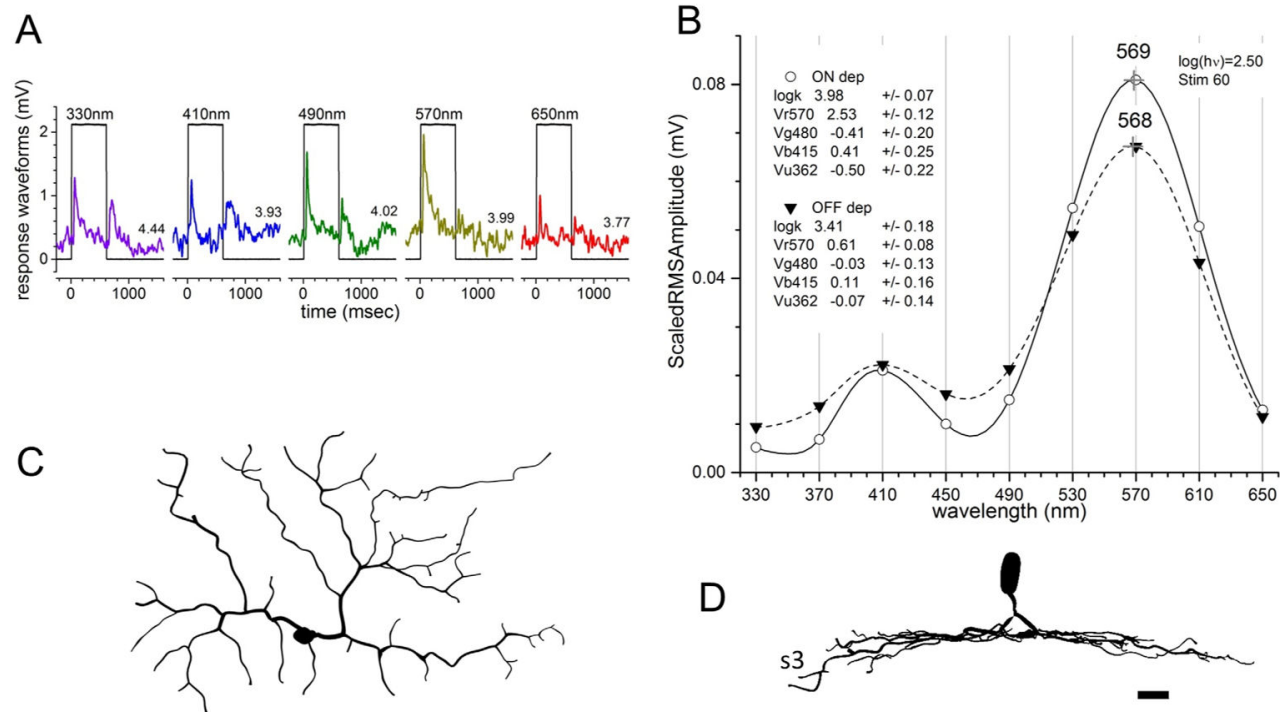

Figure 6.

ON-OFF amacrine, monostratified in s3 of sublamina a. A: Wavelengths from $330 \mathrm{~nm}$ to $650 \mathrm{~nm}$ transiently depolarize (dep) this neuron following both stimulus onset and offset. Numbers above each trace are the stimulus irradiances in $\log \left(h v \cdot \mu \mathrm{m}^{-2} \cdot \mathrm{s}^{-1}\right)$. Waveforms are filtered by a 33-point (16.5-msec) running average. Penetration potential, $-17 \mathrm{mV}$. B: The spectral model (Eq. 1, 60 stimuli) is fit both to onset (ON dep, open circles) and to offset (OFF dep, filled triangles) transients using RMS amplitudes during the 300-msec time segments after stimulus onset, and after the end of each stimulus. The spectral curves are interpolations (Eq. 1) of amplitudes for constant quantal stimuli at $2.5 \log \left(h v \cdot \mu \mathrm{m}^{-2} \cdot \mathrm{s}^{-1}\right)$. Fit parameters $( \pm \mathrm{SE})$ indicate that depolarization from red cones is significant both at $\mathrm{ON}$ and at OFF ( Vr570 $\neq 0$ ), and that small hyperpolarizing influences of both green and UV cones are also significant in the ON transient (ON dep, Vg480 $\neq 0, \mathrm{Vu} 362 \neq 0$ ). C: Neurolucida reconstruction of the dendritic field. D: Rotation of the $3 \mathrm{D}$ reconstruction reveals a single plane of dendritic branching in IPL s3, next to the sublamina $a / b$ border. 6Oct2009 Cell\#3, adult GE4a. Scale bar $=10 \mu \mathrm{m}$ in $\mathrm{C}$ and D. [Color figure can be viewed at wileyonlinelibrary.com] 


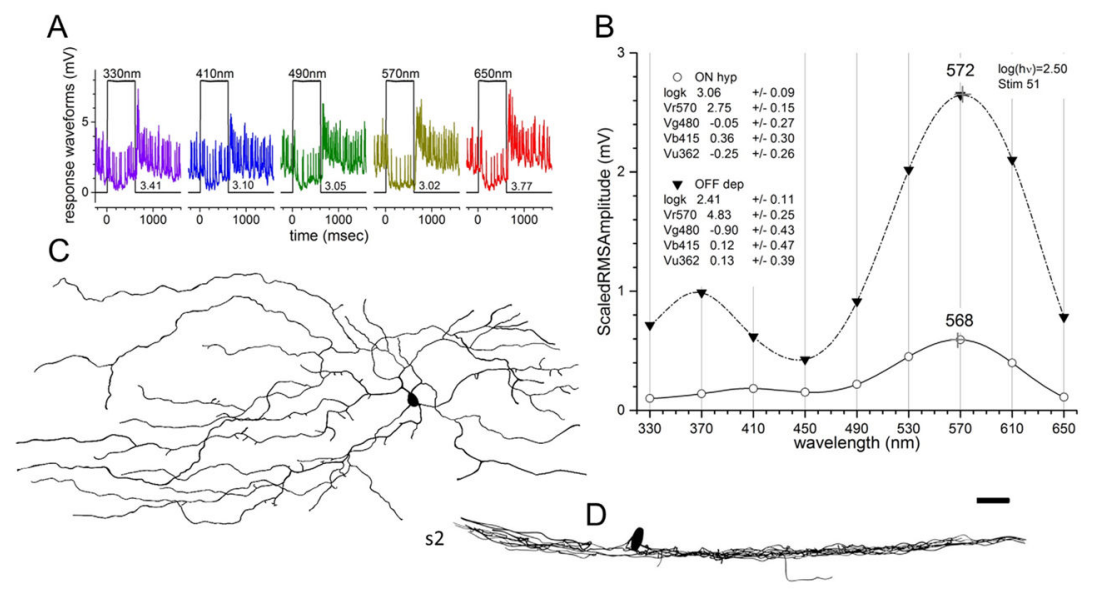

Figure 7.

Sustained OFF amacrine cell, monostratified in s2 of IPL sublamina a. A: Sustained hyperpolarization (hyp) during stimulation and transient depolarization (dep) at stimulus OFF are evoked by wavelengths from 330 to $650 \mathrm{~nm}$. This cell spontaneously fires action potentials whose frequency is modulated by light. The traces are unfiltered $(0.5 \mathrm{msec}$ sampling). Numbers below each trace are the stimulus irradiances in $\log \left(h v \cdot \mu \mathrm{m}^{-2} \cdot \mathrm{s}^{-1}\right)$. Penetration potential, $-30 \mathrm{mV}$. B: After filtering out impulse activity (101-point, 50.5-msec running average, not shown), spectral sensitivities were interpolated (Eq. 1, $2.5 \log (h v \cdot \mu \mathrm{m}$ $\left.{ }^{-2} \cdot \mathrm{s}^{-1}\right), 51$ stimuli) for the RMS hyperpolarizations during the 300-msec segment following stimulus onset (ON hyp, open circles) and for the RMS depolarizations during the $300 \mathrm{msec}$ following stimulus offset (OFF dep, filled triangles). Fit parameters $( \pm$ SE) indicate that red cone signals $(\mathrm{Vr} 570 \neq 0$ ) significantly hyperpolarize the cell at onset, and depolarize the cell at offset. Signals from other cones were not significant. Both hyperpolarization and depolarization are plotted as positive amplitudes. C: Neurolucida reconstruction of the cell's dendritic field. D: Rotation of the 3D reconstruction reveals a single plane of dendritic branching in IPL s2 (sublamina a). 8Jul2009 Cell \#1, adult GE4a. Scale bar $=20 \mu \mathrm{m}$ in C and D. [Color figure can be viewed at wileyonlinelibrary.com] 


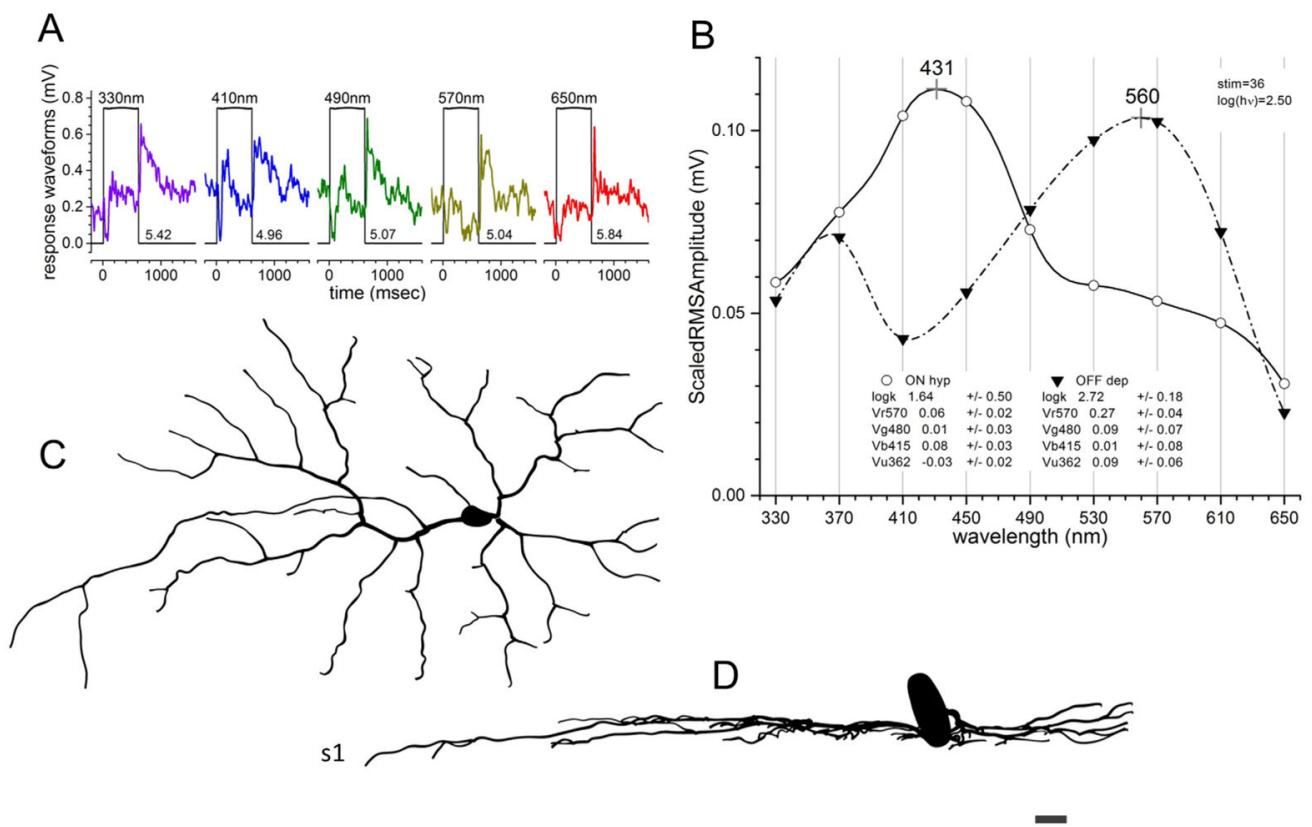

Figure 8.

Transient OFF amacrine cell, monostratified in s1 of IPL sublamina a. A: Light response waveforms consist of transient hyperpolarization (hyp) at stimulus onset followed by delayed depolarization during the stimulus, and finally, transient depolarization (dep) at stimulus offset. These three components are evoked by all wavelengths $(330-650 \mathrm{~nm})$. Numbers adjacent to the photocell offset are the stimulus irradiances in $\log \left(h \nu \cdot \mu \mathrm{m}^{-2} \cdot \mathrm{s}^{-1}\right)$. Waveforms are filtered by a 33-point $(16.5$-msec) running average. Penetration potential, -4 $\mathrm{mV}$. B: Spectral sensitivities for RMS amplitudes of the ON hyperpolarization (ON hyp, 0$100 \mathrm{msec}$, open circles), and OFF depolarizing transient (OFF dep, 600-900 msec, filled triangles) are interpolated (Eq. 1) for constant quantal stimuli of $2.5 \log \left(h \nu \cdot \mu \mathrm{m}^{-2} \cdot \mathrm{s}^{-1}\right)$. Onset and offset components are spectrally distinct. The fit parameters $( \pm \mathrm{SE})$ indicate that significant red $(\mathrm{Vr} 570 \neq 0)$ and blue $(\mathrm{Vb} 415 \neq 0)$ signals contribute to the $\mathrm{ON}$ hyperpolarizing transient, but only red $(\mathrm{Vr} 570 \neq 0)$ signals contribute to the transient OFF depolarization. C: Neurolucida reconstruction of the dendritic field. D: Rotation of the 3D reconstruction reveals a single plane of dendritic branching in IPL s1, at the amacrine border of sublamina a. 9Dec2009 Cell\#8, adult GE4a. Scale bar $=10 \mu \mathrm{m}$ in C and D. [Color figure can be viewed at wileyonlinelibrary.com] 

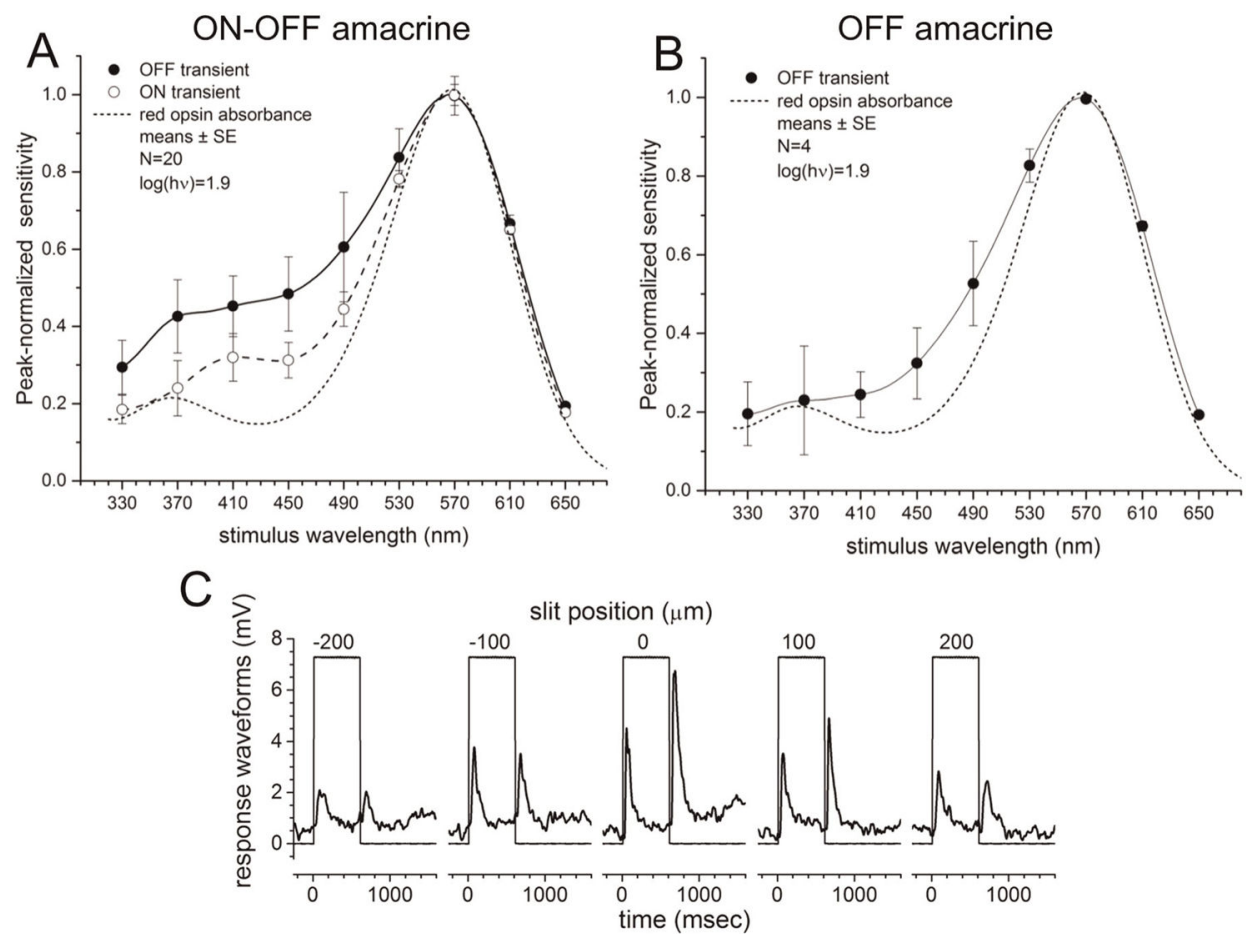

Figure 9.

Properties of transient depolarizations in $\mathrm{ON}-\mathrm{OFF}$ and $\mathrm{OFF}$ amacrine cells. A: Normalized spectra at $\mathrm{ON}$ and at OFF for $20 \mathrm{ON}-\mathrm{OFF}$ and ON-OFF-Sustained amacrine cells.

Physiologies from three stratification patterns are averaged together. Stimulus onset spectrum (open circles); stimulus offset spectrum (filled circles). Both ON and OFF components are dominated by signals from red opsin absorbance (dotted line, the Ar570 template; Hughes et al., 1998). B: Normalized spectra for offset depolarizations (filled circles) from four s1 or s2, OFF sustained and OFF transient amacrine cells. The mean spectrum for OFF-depolarization closely matches the red cone template absorbance (dotted line). In A and B, spectra are means and SEs of normalized spectral fits (Eq. 1), to RMS onset or offset amplitudes for individual-cell stimulus-response datasets. The time segments for RMS amplitude calculation are the first $300 \mathrm{msec}$ of stimulation for ON responses, and the $300 \mathrm{msec}$ following stimulation for OFF responses. The fixed-irradiance for these spectra is $1.9 \log \left(\mathrm{h} \nu \cdot \mu \mathrm{m}^{-2} \cdot \mathrm{s}^{-1}\right)$. C: Slit-mapped receptive field of ON-OFF amacrine cell. $\mathrm{ON}-\mathrm{OFF}$ responses occur at all slit positions. Slit width, $85 \mu \mathrm{m}, 610 \mathrm{~nm}, 4.8 \log \left(\mathrm{h} v \cdot \mu \mathrm{m}^{-2} \cdot \mathrm{s}\right.$ $-1)$. Same cell as in Figure 5. 
A

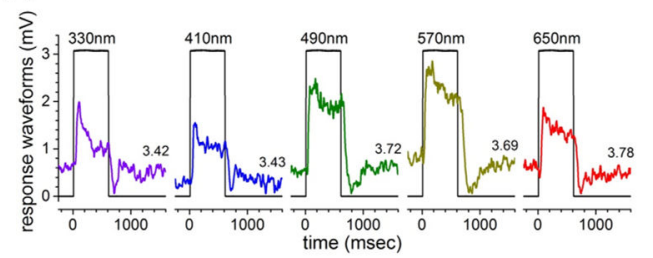

C

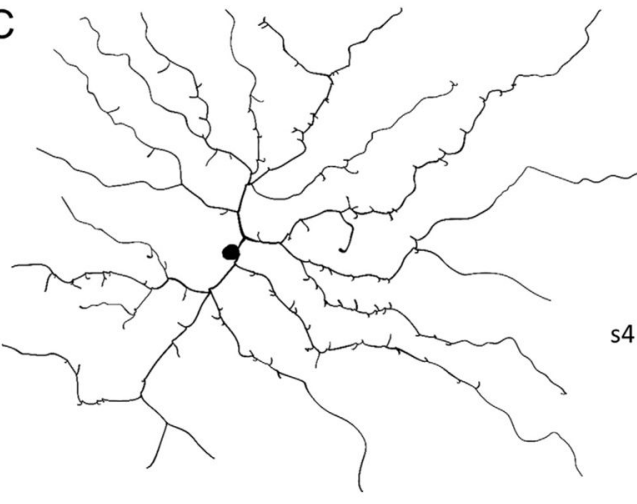

B

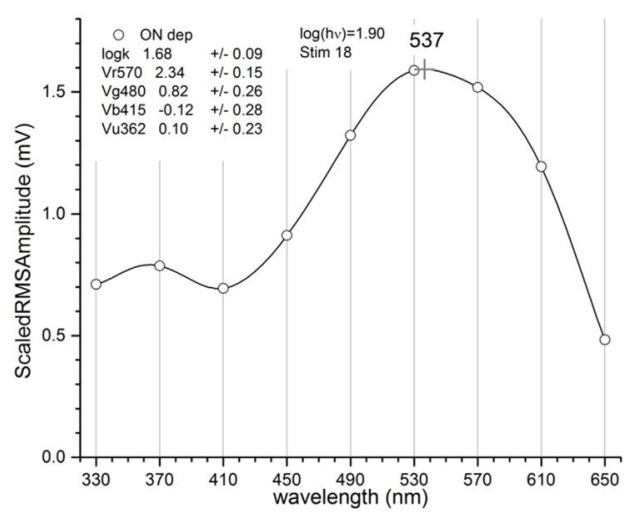

Figure 10.

Sustained ON amacrine cell, monostratified in $\mathrm{s} 4$ of IPL sublamina $b$. A: Light stimulation evokes sustained depolarization (dep), without transient depolarization at offset, for all wavelengths (330-650 nm). Numbers above each trace are the stimulus irradiances in $\log \left(h v \cdot \mu \mathrm{m}^{-2} \cdot \mathrm{s}^{-1}\right)$. Waveforms are filtered by a 33-point $(16.5$-msec) running average. Penetration potential, $-15 \mathrm{mV}$. B: Spectral sensitivities are fit (Eq. 1) to the RMS amplitudes during the initial $300 \mathrm{msec}$ in each of the 18 stimuli. The spectral curve (ON dep, open circles) is an interpolation (Eq. 1) of the response amplitudes for constant quantal stimuli at $1.9 \log \left(h v \cdot \mu \mathrm{m}^{-2} \cdot \mathrm{s}^{-1}\right)$. The fit parameters $( \pm \mathrm{SE})$ indicate that both red and green cones significantly depolarize the cell $(\mathrm{Vr} 570 \neq 0, \mathrm{Vg} 480 \neq 0)$. C: Neurolucida reconstruction of the flat-mount dendritic spread for this cell. D: Rotation of this 3D reconstruction reveals a single plane of dendritic branching in IPL $\mathrm{s} 4$, in distal sublamina $b$. The curvature of the dendritic plane results from a retina fold. 04Sept2009 \#10, adult GE4a. Scale bar $=10 \mu \mathrm{m}$ in $\mathrm{C}$ and D. [Color figure can be viewed at wileyonlinelibrary.com] 


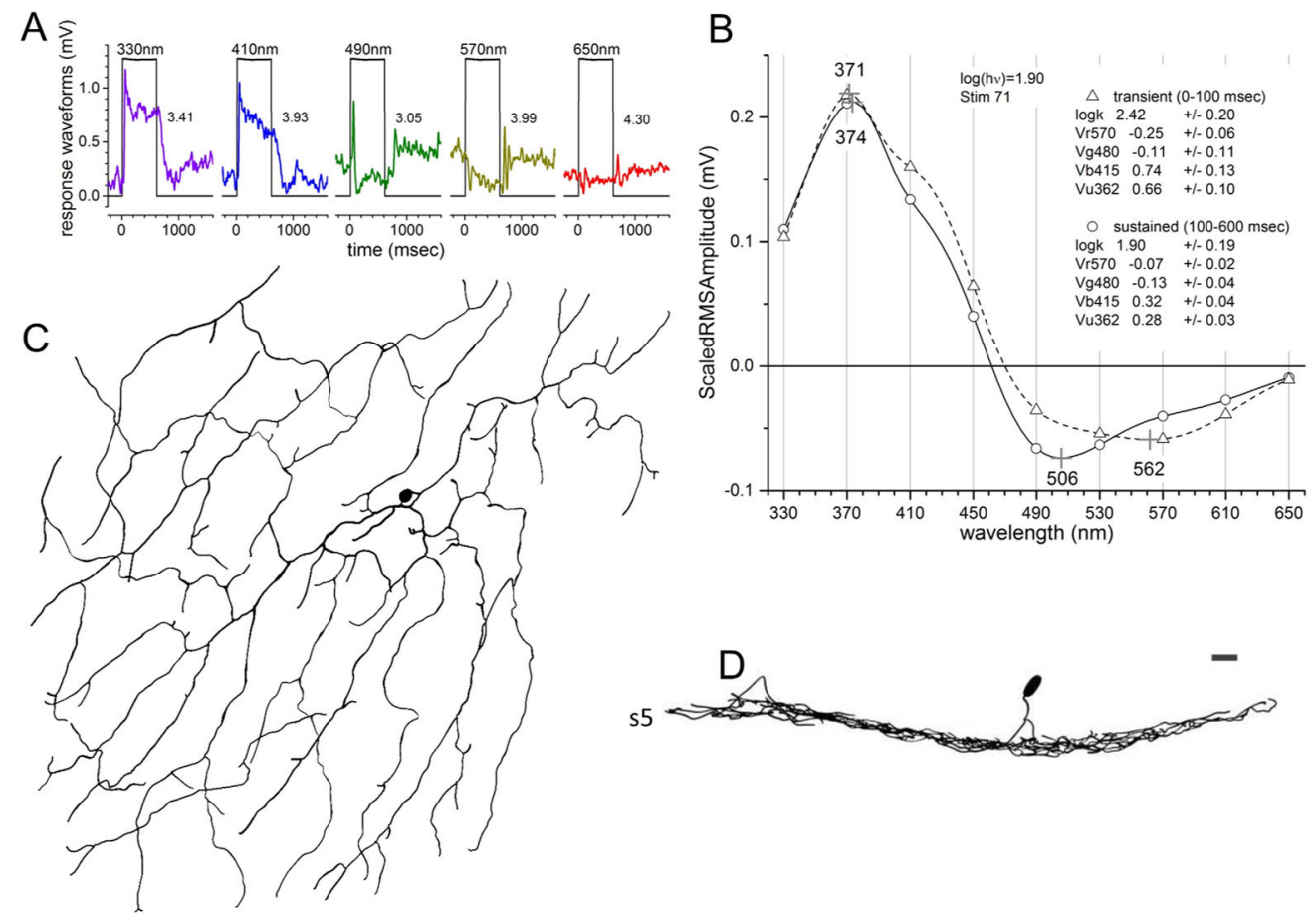

Figure 11.

UV depolarized, spectrally biphasic amacrine cell, monostratified in s5 of IPL sublamina $b$.

A: Sustained depolarization occurs with short and UV wavelengths (330-410 nm).

Midspectral stimuli evoke transient depolarization followed by sustained hyperpolarization $(490 \mathrm{~nm})$. Numbers above each trace are the stimulus irradiances in $\log \left(h \nu \cdot \mu \mathrm{m}^{-2} \cdot \mathrm{s}^{-1}\right)$.

Waveforms are filtered by a 33-point (16.5-msec) running average. Penetration potential, -8 $\mathrm{mV}$. B: Spectral sensitivities are fit (Eq. 1, 71 stimuli) to the RMS amplitudes of the onset transients (0-100 msec, open triangle) or the sustained response (100-600 msec, open circle). In the transient, UV and blue cone signals are depolarizing (Vu362 $\neq 0, \mathrm{Vb} 415 \neq 0$ ), while red cone signals are hyperpolarizing $(\operatorname{Vr} 570 \neq 0)$. The sustained phase is similar, except that green cone hyperpolarization $(\mathrm{Vg} 480 \neq 0)$ is evident, shifting the peak hyperpolarization from 562 to $506 \mathrm{~nm}$. The green cone hyperpolarizing signal appears delayed. C: Neurolucida reconstruction of this cell. D: Rotation of the 3D reconstruction reveals a single plane of dendritic branching in IPL s5, in the middle of sublamina $b$. 10Nov2009 Cell\#1, adult WT. Scale bar $=10 \mu \mathrm{m}$ in C and D. [Color figure can be viewed at wileyonlinelibrary.com] 
A
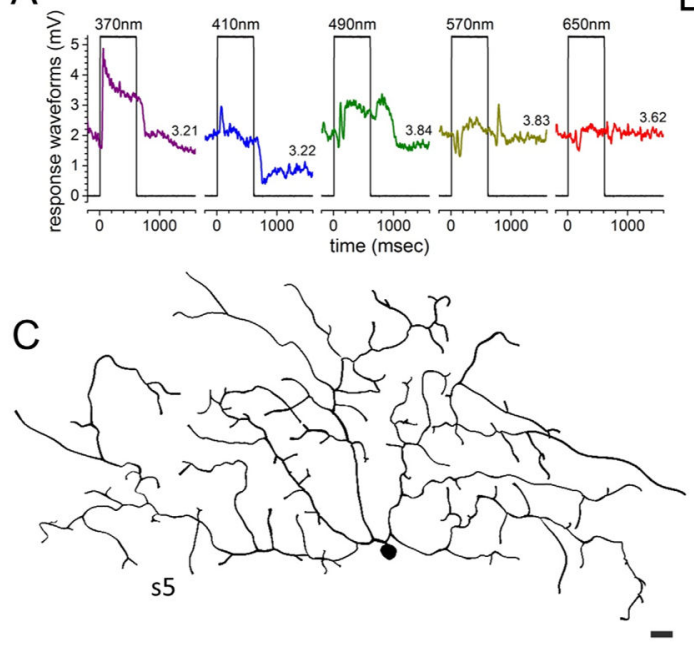

B

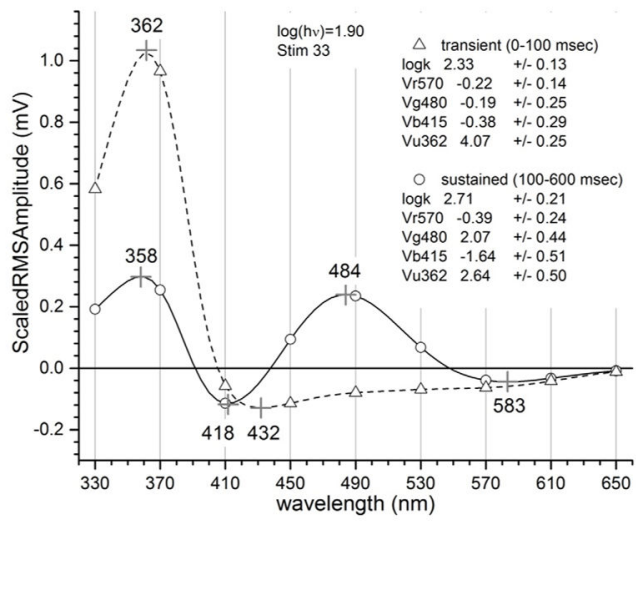

Figure 12.

UV depolarized, spectrally triphasic amacrine cell, monostratified in s5 of IPL sublamina $b$. A: Sustained depolarization occurs with UV $(370 \mathrm{~nm})$ and mid spectral $(490 \mathrm{~nm})$ stimuli. Depolarization is weak at other wavelengths. Numbers above each trace are the stimulus irradiances in $\log \left(h v \cdot \mu \mathrm{m}^{-2} \cdot \mathrm{s}^{-1}\right)$. Waveforms are filtered by a 33-point $(16.5-\mathrm{msec})$ running average. Penetration potential, $-9 \mathrm{mV}$. B: Spectral sensitivities are fit (Eq. 1, 33 stimuli) to either the onset transient (0-100 msec, open triangles) or to the sustained response (100-600 msec, open circles). In the analysis, onset transients consist of depolarizing signals arising from UV cones (Vu362 $\neq 0$ ). In the sustained component, signals arising from both UV and green cones depolarize the cell ( Vu362 $\neq 0, \mathrm{Vg} 480 \neq 0$ ), while blue cone signals are hyperpolarizing $(\mathrm{Vb} 415 \neq 0$ ). $\mathbf{C}$ : Neurolucida projection of the reconstructed cell. Examination of the image stack reveals a single plane of dendritic branching in IPL s5, in the middle of sublamina $b$. 14Nov2007 Cell\#3, adult WT. Scale bar $=10 \mu \mathrm{m}$ in C. [Color figure can be viewed at wileyonlinelibrary.com] 
A

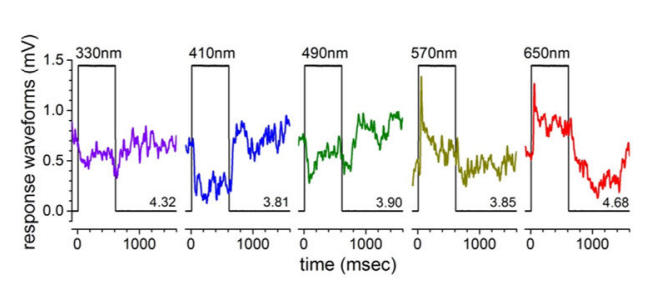

C

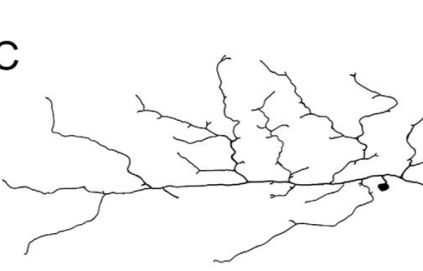

B

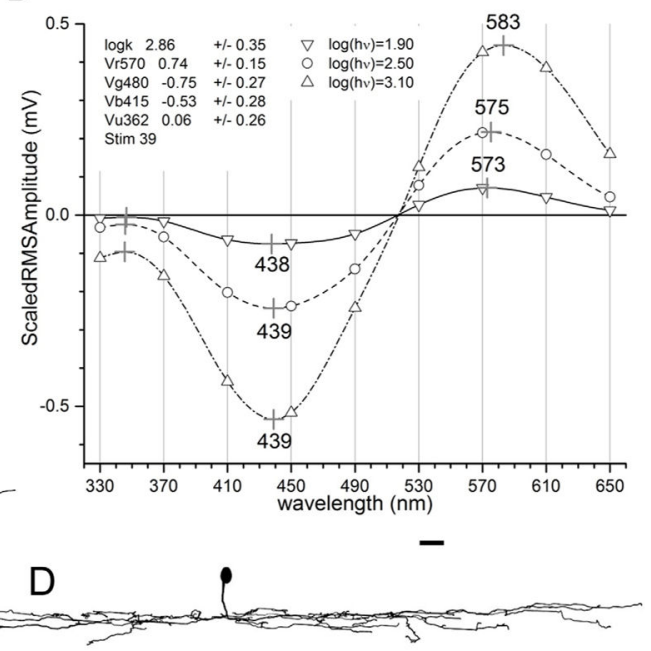

Figure 13.

Long wavelength depolarized, spectrally biphasic amacrine cell, monostratified in s5 of IPL sublamina $b$. A: Transient depolarization is seen with red $(650 \mathrm{~nm})$ or yellow $(570 \mathrm{~nm})$ stimuli, but hyperpolarization is evoked for mid and short wavelength stimuli (490 and 410 $\mathrm{nm}$, respectively). Numbers below each trace are the stimulus irradiances in $\log \left(h v \cdot \mu \mathrm{m}^{-2} \cdot \mathrm{s}\right.$ $\left.{ }^{-1}\right)$. Waveforms are filtered by a 33-point $(16.5$-msec) running average. Penetration potential, $-12 \mathrm{mV}$. B: Spectral sensitivities are fit (Eq. 1) to the RMS amplitudes during the initial 300 msec in each of the 39 stimuli. The three spectral curves of increasing amplitude are Equation 1 interpolations of the response amplitudes for constant quantal stimuli at 1.9, 2.5, or $3.1 \log \left(h v \cdot \mu \mathrm{m}^{-2} \cdot \mathrm{s}^{-1}\right)$. The fit parameters $( \pm \mathrm{SE})$ suggest that depolarization from red cones is significant $(\operatorname{Vr} 570 \neq 0$ ), while hyperpolarization is driven by both green and blue cones $(\mathrm{Vg} 480 \neq 0, \mathrm{Vb} 415 \neq 0)$. C: An elongate dendritic field is seen in Neurolucida reconstruction of this cell. D: Rotation of the 3D reconstruction reveals a single dendritic branching plane in IPL s5 (sublamina $b$ ). 20May2010 Cell\#6, adult GE4a. Scale bar $=20 \mu \mathrm{m}$ in $\mathrm{C}$ and $\mathrm{D}$. [Color figure can be viewed at wileyonlinelibrary.com] 


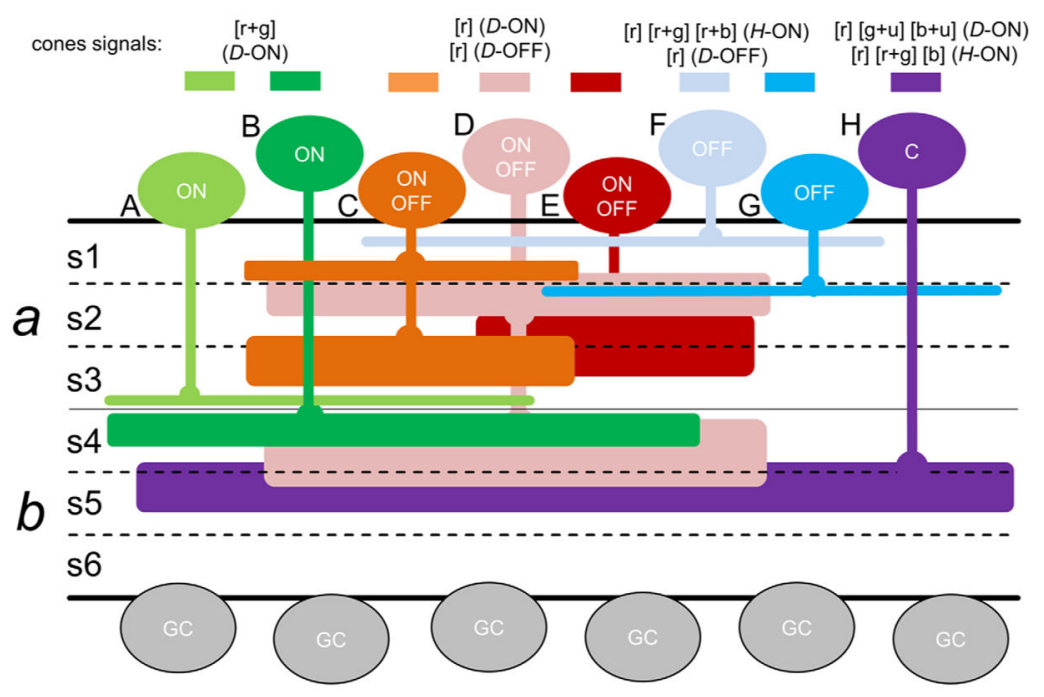

Figure 14.

IPL branching patterns of electrophysiologically grouped zebrafish amacrine cells. A,B: ON physiologies are recorded from monostratified cells arborizing mainly in s4, but occasionally in s3. C,D: ON-OFF and ON-OFF-Sustained physiologies almost always arise from bistratified dendritic patterns. Most (D) branch in both sublamina $a$ (s1 or s2) and sublamina $b$ (s4 or s5). Others (C) are bistratified within sublamina $a$, occupying s1, and either s2 or s3. E: Rarely, an ON-OFF type is monostratified in s2 or s3, sublamina a. F,G: OFF physiologies come from cells monostratified in either s1 or s2. H: C-type physiologies are depolarized by some wavelengths and hyperpolarized by others, with a variety of spectral patterns. C-type physiology occurs mainly in s5-monostratified amacrines of sublamina $b$. Ganglion cell (GC) bodies occupy the s6 border of the IPL. The breadth of amacrine stratification bands represent the depth range within the IPL occupied by the dendrites of the physiological groups. The "cone signals" legend (above) gives the polarities and types of cone inputs (r, g, b, u) in each waveform/stratification group. $D$ and $H$ stand for depolarizing or hyperpolarizing signals at onset $(\mathrm{ON})$ or offset (OFF). [Color figure can be viewed at wileyonlinelibrary.com] 


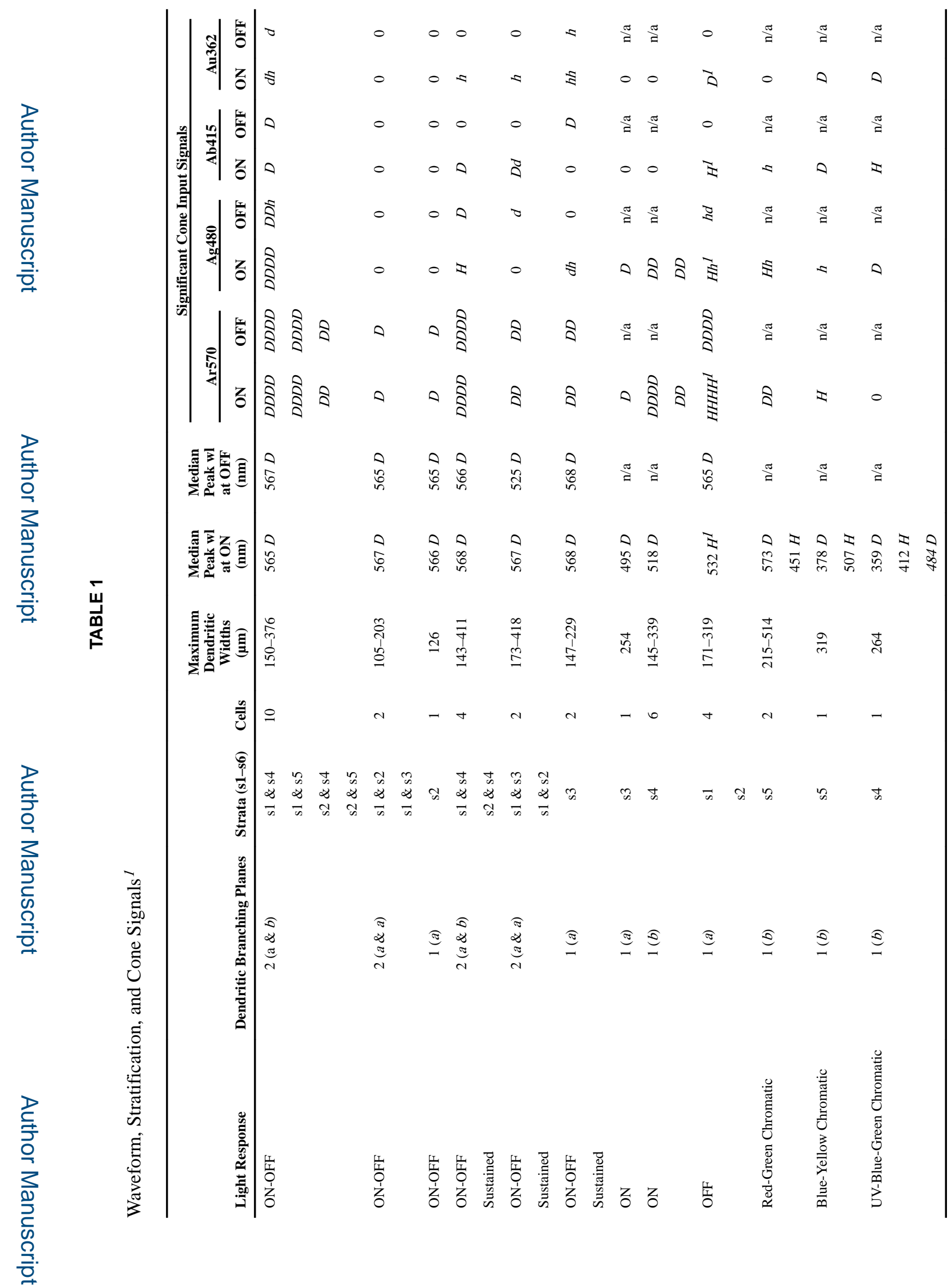

J Comp Neurol. Author manuscript; available in PMC 2018 August 13. 


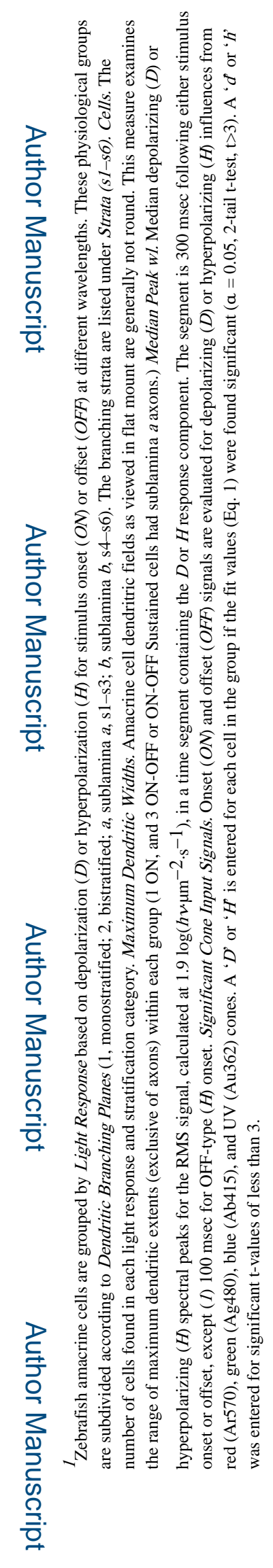

J Comp Neurol. Author manuscript; available in PMC 2018 August 13. 